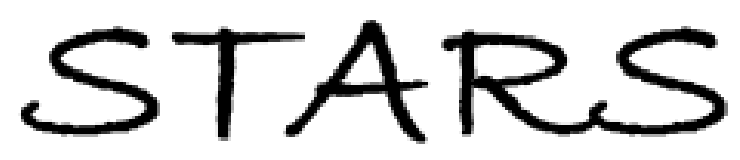

University of Central Florida

STARS

$1-1-2015$

\title{
Atomic-scale simulation of space weathering in olivine and orthopyroxene
}

\author{
Abrar H. Quadery \\ University of Central Florida \\ Shaun Pacheco \\ University of Central Florida \\ Alan Au \\ University of Central Florida \\ Natalie Rizzacasa \\ University of Central Florida \\ Joshua Nichols \\ University of Central Florida \\ Find similar works at: https://stars.library.ucf.edu/facultybib2010 \\ University of Central Florida Libraries http://library.ucf.edu \\ See next page for additional authors
}

This Article is brought to you for free and open access by the Faculty Bibliography at STARS. It has been accepted for inclusion in Faculty Bibliography 2010 s by an authorized administrator of STARS. For more information, please contactSTARS@ucf.edu.

\section{Recommended Citation}

Quadery, Abrar H.; Pacheco, Shaun; Au, Alan; Rizzacasa, Natalie; Nichols, Joshua; Le, Timothy; Glasscock, Cameron; and Schelling, Patrick K., "Atomic-scale simulation of space weathering in olivine and orthopyroxene" (2015). Faculty Bibliography 2010s. 6768.

https://stars.library.ucf.edu/facultybib2010/6768

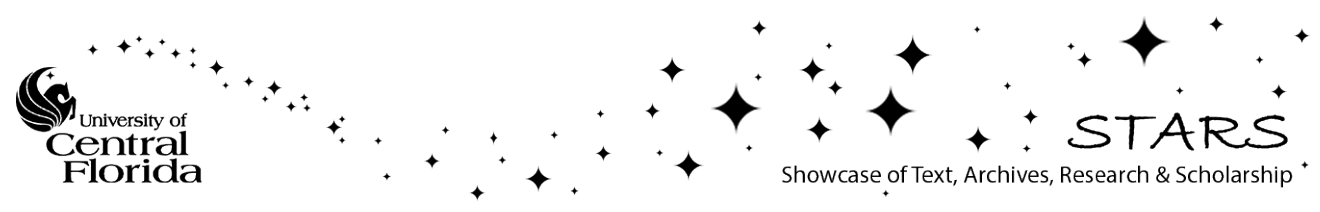




\section{Authors}

Abrar H. Quadery, Shaun Pacheco, Alan Au, Natalie Rizzacasa, Joshua Nichols, Timothy Le, Cameron Glasscock, and Patrick K. Schelling 


\section{Journal of Geophysical Research: Planets}

\author{
RESEARCH ARTICLE \\ 10.1002/2014JE004683 \\ Key Points: \\ - We develop interatomic potentials for \\ olivine and orthopyroxene \\ - The annealing behavior of Frenkel \\ defects in the two minerals is \\ different \\ - Differences in defects evolution lead \\ to different space weathering rates
}

Correspondence to:
P. K. Schelling,
Patrick.Schelling@ucf.edu

\section{Citation:}

Quadery, A. H., S. Pacheco, A. Au, N. Rizzacasa, J. Nichols, T. Le, C. Glasscock, and P. K. Schelling (2015), Atomic-scale simulation of space weathering in olivine and orthopyroxene, J. Geophys. Res. Planets, 120, 643-661, doi:10.1002/2014JE004683.

Received 28 JUN 2014 Accepted 9 FEB 2015 Accepted article online 13 FEB 2015 Published online 10 APR 2015

\section{Atomic-scale simulation of space weathering in olivine and orthopyroxene}

\author{
Abrar H. Quadery ${ }^{1}$, Shaun Pacheco ${ }^{1}$, Alan Au ${ }^{1}$, Natalie Rizzacasa ${ }^{1}$, Joshua Nichols ${ }^{1}$, \\ Timothy Le ${ }^{1}$, Cameron Glasscock ${ }^{1}$, and Patrick K. Schelling ${ }^{1,2}$ \\ ${ }^{1}$ Department of Physics, University of Central Florida, Orlando, Florida, USA, ${ }^{2}$ Advanced Material Processing and Analysis \\ Center, University of Central Florida, Orlando, Florida, USA
}

Abstract Classical molecular dynamics was used to study the annealing of anion and cation Frenkel defects in olivine and orthopyroxene minerals. While it was found that for both minerals, reorganization of the $\mathrm{Si}-\mathrm{O}$ bonds, often accompanied by large Si displacements, occurs to maintain the fourfold coordination of the $\mathrm{SiO}_{4}$ tetrahedra, important differences are observed in their annealing behavior. Specifically, cation defects are substantially more mobile in olivine than in orthopyroxene leading to rapid annihilation of cation Frenkel defects and formation of extended defects in olivine. By contrast, the diffusion rate of anion defects in orthopyroxene is much higher than that in olivine and also exhibits large anisotropy. Consequently, it was found that diffusion in orthopyroxene occurs without significant annihilation of anion Frenkel defects or trapping of anion interstitials or vacancies into clusters. The results obtained here are discussed in the context of space weathering of olivine and orthopyroxene. Specifically, two important observations are made which may explain previous experimental results. First, ion irradiation experiments that show reduced tolerance for radiation damage in orthopyroxene may be explained by the rapid, one-dimensional anion mobility which prevents healing of the lattice. Second, laser heating experiments which show that orthopyroxene has enhanced tolerance to reduction and the evolution of nanophase Fe inclusions could be due to the observed rapid anion diffusion in orthopyroxene, which might allow the bulk to act as a reservoir for the surface.

\section{Introduction}

While computational studies of radiation damage are quite common, the application of atomic-scale simulation methodology has generally focused on either elemental metals or semiconductors [Stoller, 2012]. Recently, there have been significant efforts to use atomic-scale simulation to study the evolution of radiation damage in binary and more complex oxides including $\mathrm{MgO}$ [Aidhy et al., 2009a], $\mathrm{UO}_{2}$ [Aidhy et al., 2009b, 2011], $\mathrm{CeO}_{2}$ [Aidhy et al., 2011], $\mathrm{ZrO}_{2}$ [Zarkadoula et al., 2014], and $\mathrm{ZrCaTi}_{2} \mathrm{O}_{7}$ [Yang et al., 2014]. The general picture which has emerged is that radiation damage generates Frenkel defects (interstitial-vacancy pairs), although more severe damage can lead to amorphization [Golubov, 2012]. Annealing in radiation damaged material takes place through a number of processes, which include point defect migration, recombination of vacancies and interstitials, and the formation of extended defect structures, including dislocations and point defect clusters. One important result that has been shown with atomic-scale simulation is that extended defect clusters act as traps for point defects, thereby inhibiting further migration and vacancy-interstitial recombination [Aidhy et al., 2009a, 2009b, 2011].

There have been very few computational studies relevant for understanding radiation damage in silicate minerals [Kubota et al., 2003]. In the present article, the primary motivation for modeling radiation damage in silicate minerals is to understand the solid-state effects of energetic particles, mostly $\mathrm{H}^{+}$and $\mathrm{He}^{+}$ions, from the solar wind. Solar wind particles are an important contributing source to the space weathering of airless bodies in the solar system, including the Moon, Mercury, and asteroids [Cassidy and Hapke, 1975; Hapke, 2001; Chapman, 2004]. Transmission electron microscopy (TEM) studies of lunar materials demonstrate that the characteristic amorphous rims are due to a combination of radiation damage, sputtering, and impact vapor deposition [Hapke, 2001; Keller and McKay, 1993, 1997; Christoffersen et al., 1996]. Another characteristic feature of space weathered material is the presence of nanometer sized elemental Fe inclusions, often termed as nanophase iron $\left(\mathrm{npFe}^{0}\right)$ that result from strong reduction. The generally accepted view is that the $n p F e^{0}$ is primarily produced as reduced impact vapors condense on 


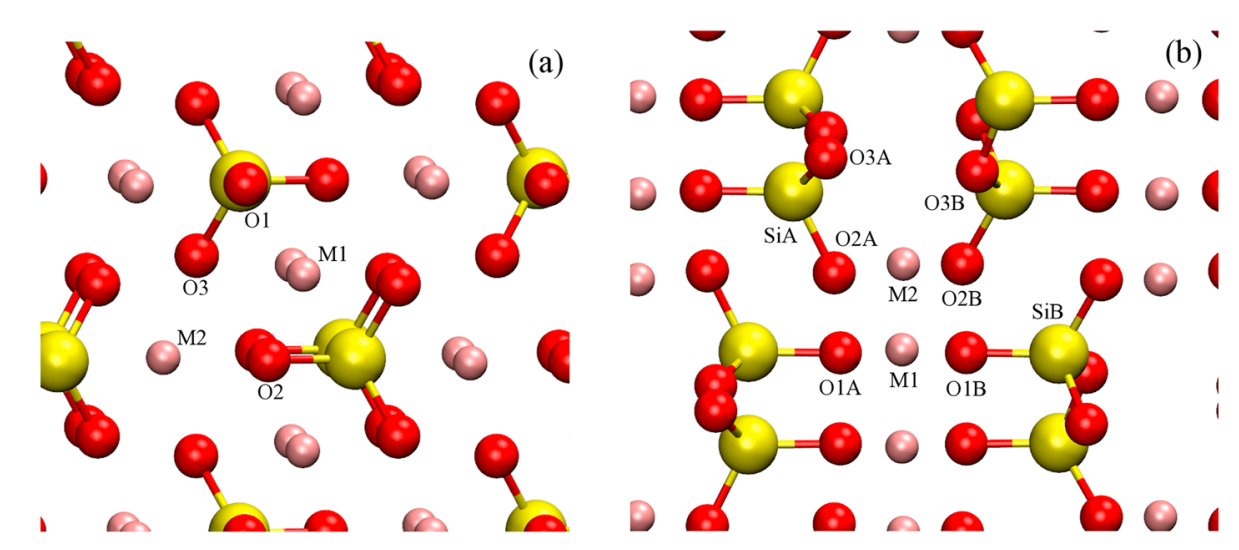

Figure 1. Crystal structure of (a) olivine and (b) orthopyroxene. Here grey, yellow, and red spheres represent Mg/Fe sites, $\mathrm{Si}$ sites, and $\mathrm{O}$ sites, respectively. In olivine, the $\mathrm{SiO}_{4}$ tetrahedra are isolated, whereas in pyroxene each tetrahedron shares two of its oxygen with two neighboring tetrahedra to form a silicate chain along $c$ axis of the crystal.

grain surfaces [Hapke, 2001; Keller and McKay, 1993, 1997; Christoffersen et al., 1996]. However, recent analysis of samples returned from the asteroid Itokawa suggests that beneath a layer of material deposited from vaporized material, there exists a layer which shows evidence of partial amorphization and reduction from radiation damage [Noguchi et al., 2014; Thompson et al., 2014]. The presence of $n p F e^{0}$ has been directly observed in both layers, which gives direct evidence that $n \mathrm{nFe}^{0}$ can be produced by radiation damage as well as condensation of reduced impact vapor. Hence, the results from Itokawa present a contrast from the accepted mechanism for $\mathrm{npFe}^{0}$ in lunar samples, although several open questions remain. It may be, for example, that reduction and $\mathrm{npFe}^{0}$ occur due to preferential oxygen sputtering from the solar wind.

However, it is not yet understood under what conditions solar wind irradiation might compete with impact vapor deposition as a mechanism to generate $\mathrm{npFe}^{0}$. In short, the mechanisms by which radiation damage can result in amorphization and $\mathrm{npFe} \mathrm{e}^{0}$ inclusions remain incompletely understood.

In this paper, we report simulated annealing of Frenkel defects in olivine $\left(\mathrm{Mg}_{2} \mathrm{SiO}_{4}\right.$ and $\left.\mathrm{Fe}_{2} \mathrm{SiO}_{4}\right)$ and orthopyroxene $\left(\mathrm{MgSiO}_{3}\right.$ and $\left.\mathrm{FeSiO}_{3}\right)$ at $T=1200 \mathrm{~K}$ using molecular dynamics (MD) simulation. The simulations were based on an empirical pair potential optimized to describe the relevant minerals. It is shown that defect diffusion and clustering occur by mechanisms which act to maintain the coordination of the $\mathrm{SiO}_{4}$ tetrahedra. While this aspect is common to both olivine and orthopyroxene, there are some clear and striking differences. Specifically, cation diffusion occurs more readily in olivine than in orthopyroxene. As a result, cation Frenkel defects heal more rapidly in olivine minerals. The rapid cation diffusion in olivine leads to rapid formation of point defect clusters, in which point defects get trapped. On the other hand, diffusion of anion defects, especially oxygen interstitials, occurs more rapidly in orthopyroxene than olivine. However, it is found that the diffusion of anion interstitials in orthopyroxene is highly anisotropic, leading to long chains of anion vacancies and interstitials. As a result, despite the fact that anion interstitials migrate very rapidly in orthopyroxene, the highly anisotropic nature of the diffusion inhibits both recombination with anion vacancies and trapping into point defect clusters. It is also found that split interstitials and split vacancies are important defect states.

The results are discussed in terms of potential relevance to space weathering phenomena. The particular focus is on comparison to existing radiation damage experiments performed on enstatite and olivine samples [Christoffersen and Keller, 2011; Wang et al., 1993a, 1993b, 1998; Carrez et al., 2002]. Irradiation experiments using heavy ions by Christoffersen and Keller [2011] showed a similar critical radiation dose for amorphization in olivine and enstatite, although olivine was found to be slightly more resistant to amorphization. On the other hand, Wang et al. [1998] found that olivine required about 4 times higher radiation dose than enstatite to get amorphized, suggesting olivine might be more radiation resistant. In Carrez et al. [2002], radiation with $\mathrm{He}^{+}$ions consistent with solar wind conditions was used to determine the critical amorphization dose in olivine. However, no comparable experiment relevant for solar wind conditions exists for enstatite. In another spectrum of experimental simulations of space weathering, where pulsed laser heating had been used to effect micrometeorite impacts, it has been observed that spectral alterations take place more readily in olivine than in orthopyroxene [Yamada et al., 1999; Sasaki et al., 2001; Sasaki and 
Table 1. Parameters for the Empirical Pair Potentials Described by Equation (1); Parameters Optimized for Olivine and Orthopyroxene

\begin{tabular}{lcccc} 
Pair & $D(\mathrm{eV})$ & $a\left(\AA^{-1}\right)$ & $r_{0}(\AA)$ & $C\left(\mathrm{eV} \AA^{12}\right)$ \\
\hline $\mathrm{Mg}-\mathrm{O}$ & 0.123583 & 2.045583 & 2.424824 & 5.0 \\
$\mathrm{Fe}-\mathrm{O}$ & 0.064948 & 1.888936 & 2.644110 & 2.0 \\
$\mathrm{Si}-\mathrm{O}$ & 0.443427 & 1.758024 & 2.081625 & 1.0 \\
$\mathrm{O}-\mathrm{O}$ & 0.042323 & 1.311417 & 3.762599 & 22.0 \\
\hline
\end{tabular}

Kurahashi 2004]. However, there has been essentially no effort to understand what might be occurring at the atomic scale. The results presented here lead to some insights that might explain how olivine differs from orthopyroxene, both in the context of resistance to radiation damage and the formation of nanophase Fe in pulsed laser experiments.

\section{Atomic-Scale Simulation Methodology}

\subsection{Pairwise Potential Development}

Both olivine and orthopyroxene are orthorhombic crystals [Birle et al., 1968; Hazen, 1976; Sueno et al., 1976]. The mineral composition generally includes both $\mathrm{Mg}$ and $\mathrm{Fe}$, along with other elements at lower concentrations, resulting in many different compositions. The composition of olivine can vary continuously between forsterite $\mathrm{Mg}_{2} \mathrm{SiO}_{4}$ and fayalite $\mathrm{Fe}_{2} \mathrm{SiO}_{4}$. Similarly, orthopyroxene can have a composition that varies between the end-members orthoenstatite $\mathrm{MgSiO}_{3}$ and orthoferrosilite $\mathrm{FeSiO}_{3}$.

Representation of the crystal structures of olivine and orthopyroxene are shown in Figure 1. The basic building blocks of olivine are two distinct $\mathrm{MO}_{6}$ octahedral units and one $\mathrm{SiO}_{4}$ tetrahedral unit. The $\mathrm{Mg} / \mathrm{Fe}$ ions occupy the $\mathrm{M} 1$ or $\mathrm{M} 2$ octahedral sites. The $\mathrm{M} 1$ and $\mathrm{M} 2$ sites are characterized by different $\mathrm{M}-\mathrm{O}$ bond lengths [Hazen, 1976]. In the orthopyroxene structure, as with the olivine, there are two distinct Mg/Fe sites labeled M1 and M2. In addition, the orthopyroxene structure has two distinct Si sites SiA and SiB. There are six distinct $\mathrm{O}$ sites. The sharing of oxygen ions by neighboring tetrahedra is a characteristic of orthopyroxene. Specifically, $\mathrm{SiO}_{4}$ tetrahedra are linked together by shared oxygen ions, resulting in tetrahedral chains along the $c$ axis. Later on, the orientation of the chains will be shown to be connected to strong anisotropy in anion diffusion.

The empirical pair potentials $\left(u_{i j}\right)$ used in this work are based on the standard Morse potential, Coulombic interactions with fixed point charges, and a short-ranged $1 / r^{12}$ repulsive potential, and have the following form:

$$
u_{i j}\left(r_{i j}\right)=\frac{q_{i} q_{j}}{r_{i j}}+D_{i j}\left[\left(1-e^{-a_{i j}\left(r_{i j}-r_{0}\right)}\right)^{2}-1\right]+\frac{C_{i j}}{r_{i j}^{12}}
$$

Table 2. Computed Properties of Ground State Forsterite Compared With Experimental Values ${ }^{a}$

\begin{tabular}{lcccc} 
Property & Pedone et al. [2006] & Pedone et al. [2006] $(T=0 \mathrm{~K})$ & This Paper & Experiment \\
\hline $\mathrm{a}(\AA)$ & 4.8447 & 4.8454 & 4.7806 & 4.7530 \\
$\mathrm{~b}(\AA \AA)$ & 10.1875 & 10.1891 & 10.1418 & 10.1900 \\
$\mathrm{C}(\AA)$ & 6.0022 & 6.0032 & 5.9632 & 5.9780 \\
$\Omega\left(\AA^{3}\right)$ & 296.2444 & 296.3792 & 289.1169 & 289.5329 \\
$\mathrm{C}_{11}$ & 298.2 & 298.0 & 323.6 & 328.0 \\
$\mathrm{C}_{22}$ & 174.6 & 174.5 & 195.2 & 200.0 \\
$\mathrm{C}_{33}$ & 216.0 & 216.1 & 243.3 & 235.0 \\
$\mathrm{C}_{12}$ & 69.4 & 69.5 & 72.9 & 69.0 \\
$\mathrm{C}_{13}$ & 74.1 & 74.2 & 77.2 & 69.0 \\
$\mathrm{C}_{23}$ & 74.4 & 74.4 & 85.6 & 73.0 \\
$\mathrm{C}_{44}$ & 38.7 & 38.9 & 45.9 & 66.7 \\
$\mathrm{C}_{55}$ & 58.5 & 58.5 & 68.1 & 81.3 \\
$\mathrm{C}_{66}$ & 68.2 & 68.2 & 76.1 & 80.9 \\
$B$ & 119.9 & 119.9 & 132.6 & 129.5 \\
\hline
\end{tabular}

aValues in the second column are from Pedone et al. [2006]. Values in the third column were computed here using the potential reported in Pedone et al. [2006]. The bulk modulus is computed using the Reuss approximation. Elastic constants and bulk modulus are given in units of GPa. 
Table 3. Computed Properties of Ground State Orthoenstatite Compared With Experimental Values $^{\mathrm{a}}$

\begin{tabular}{lcccc} 
Property & Pedone et al. [2006] & Pedone et al. [2006] $(T=0 \mathrm{~K})$ & This Paper & Experiment \\
\hline $\mathrm{a}(\AA)$ & 18.5219 & 18.5243 & 18.3335 & 18.2350 \\
$\mathrm{~b}(\AA)$ & 8.8489 & 8.8493 & 8.7116 & 8.8180 \\
$\mathrm{c}(\AA)$ & 5.2582 & 5.2585 & 5.1820 & 5.1790 \\
$\Omega\left(\AA^{3}\right)$ & 861.8050 & 862.0083 & 827.6299 & 832.764 \\
$\mathrm{C}_{11}$ & 202.9 & 202.9 & 260.4 & 224.7 \\
$\mathrm{C}_{22}$ & 115.2 & 115.4 & 158.9 & 177.9 \\
$\mathrm{C}_{33}$ & 155.7 & 155.5 & 210.8 & 213.6 \\
$\mathrm{C}_{12}$ & 50.1 & 50.2 & 63.4 & 72.4 \\
$\mathrm{C}_{13}$ & 32.8 & 32.9 & 50.3 & 54.1 \\
$\mathrm{C}_{23}$ & 14.6 & 14.6 & 50.2 & 52.7 \\
$\mathrm{C}_{44}$ & 68.3 & 68.4 & 86.0 & 77.6 \\
$\mathrm{C}_{55}$ & 61.6 & 61.6 & 74.3 & 75.9 \\
$\mathrm{C}_{66}$ & 53.5 & 53.5 & 63.6 & 81.6 \\
$\mathrm{~B}$ & 68.4 & 68.5 & 102.5 & 107.8 \\
\hline
\end{tabular}

aValues in the second column are from Pedone et al. [2006]. Values in the third column were computed here using the potential reported in Pedone et al. [2006]. Elastic constants and bulk modulus are given in units of GPa.

This form was used by Pedone et al. [2006] who fitted the parameters to a range of binary oxides. The resulting potentials were found to provide a very good description of more complex oxides beyond the fitted ones, including olivine and orthopyroxene. To enhance the description of the experimental properties of olivine and orthopyroxene, we further refined the parameters by fitting them simultaneously to the observed lattice parameters, elastic constants, and crystal structure of forsterite $\left(\mathrm{Mg}_{2} \mathrm{SiO}_{4}\right)$, fayalite $\left(\mathrm{Fe}_{2} \mathrm{SiO}_{4}\right)$, orthoenstatite $\left(\mathrm{MgSiO}_{3}\right)$, and ferrosilite $\left(\mathrm{FeSiO}_{3}\right)$. The fitting was done using the General Utility Lattice Program (GULP) simulation code [Gale and Rohl, 2003] with electrostatic interactions computed through Ewald method. In the fitting, the parameters $C_{i j}$ associated with the repulsive interactions were not varied and were fixed at the values reported in Pedone et al. [2006]. The parameters obtained are shown in Table 1. Finally, the potentials were shifted and gradient corrected to assure smoothness and continuity at a cutoff $r_{\text {cut }}=12 \AA$.

The predictions for the $T=0 \mathrm{~K}$ properties including the lattice parameters, unit cell volume, and elastic properties are presented in Tables 2-5 along with target experimental values [Smyth and McCormick, 1995; Bass, 1995]. We also include, except in the case of ferrosilite, the results from the Pedone potential [Pedone et al., 2006]. In contrast to the calculations reported here, the values from Pedone et al. [2006]

Table 4. Computed Properties of Ground State Fayalite Compared With Experimental Values ${ }^{a}$

\begin{tabular}{lcccc} 
Property & Pedone et al. [2006] & Pedone et al. [2006] $(T=0$ K $)$ & This Paper & Experiment \\
\hline $\mathrm{a}(\AA)$ & 4.8727 & 4.8924 & 4.8508 & 4.8200 \\
$\mathrm{~b}(\AA)$ & 10.2539 & 10.2727 & 10.3386 & 10.4790 \\
$\mathrm{c}(\AA)$ & 6.0564 & 6.0837 & 6.0686 & 6.0870 \\
$\Omega\left(\AA^{3}\right)$ & 302.6050 & 305.7522 & 304.3459 & 307.4469 \\
$\mathrm{C}_{11}$ & 296.0 & 263.3 & 269.8 & 266.0 \\
$\mathrm{C}_{22}$ & 178.5 & 152.1 & 156.5 & 168.0 \\
$\mathrm{C}_{33}$ & 217.4 & 191.8 & 205.8 & 232.0 \\
$\mathrm{C}_{44}$ & 50.6 & 40.6 & 28.7 & 32.3 \\
$\mathrm{C}_{55}$ & 64.3 & 53.4 & 54.7 & 46.5 \\
$\mathrm{C}_{66}$ & 72.0 & 62.6 & 62.6 & 57.0 \\
$\mathrm{C}_{12}$ & 76.8 & 64.6 & 57.6 & 94.0 \\
$\mathrm{C}_{13}$ & 81.6 & 67.3 & 62.3 & 92.0 \\
$\mathrm{C}_{23}$ & 76.0 & 68.1 & 71.6 & 92.0 \\
$\mathrm{~B}$ & 123.7 & 107.1 & 108.6 & 134.0 \\
\hline
\end{tabular}

avalues in the second column are from Pedone et al. [2006]. Elastic constants and bulk modulus are given in units of GPa. 
Table 5. Computed Properties of Ground State Orthoferrosilite Compared With Experimental Values ${ }^{\mathrm{a}}$

\begin{tabular}{lccc} 
Property & Pedone et al. [2006] $(T=0 \mathrm{~K})$ & This Paper & Experiment \\
\hline $\mathrm{a}(\AA)$ & 18.6658 & 18.5584 & 18.4180 \\
$\mathrm{~b}(\AA)$ & 8.8512 & 8.8556 & 9.0780 \\
$\mathrm{c}(\AA)$ & 5.2640 & 5.2389 & 5.2366 \\
$\Omega\left(\AA^{3}\right)$ & 869.6935 & 860.9917 & 875.5522 \\
$\mathrm{C}_{11}$ & 208.0 & 222.2 & 198.0 \\
$\mathrm{C}_{22}$ & 133.9 & 131.6 & 136.0 \\
$\mathrm{C}_{33}$ & 179.0 & 171.6 & 175.0 \\
$\mathrm{C}_{44}$ & 70.2 & 79.7 & 59.0 \\
$\mathrm{C}_{55}$ & 60.2 & 63.1 & 58.0 \\
$\mathrm{C}_{66}$ & 54.1 & 55.5 & 49.0 \\
$\mathrm{C}_{12}$ & 65.8 & 55.4 & 84.0 \\
$\mathrm{C}_{13}$ & 51.5 & 35.4 & 72.0 \\
$\mathrm{C}_{23}$ & 43.0 & 43.1 & 55.0 \\
$\mathrm{~B}$ & 89.9 & 85.0 & 101 \\
\hline
\end{tabular}

aValues in the second column are from Pedone et al. [2006]. Elastic constants and bulk modulus are given in units of $\mathrm{GPa}$. were obtained by minimization of the free energy within the quasiharmonic approximation at room temperature. It is important to note that the experimental properties reported in Tables 2-5 were measured at room temperature. Therefore, the experimental values are best compared to calculations using free energy minimization. However, at $T=300 \mathrm{~K}$, thermal expansion represents a small effect. From the results in Tables 2-5 for the Pedone potential, it is surprising that the $T=0 \mathrm{~K}$ results are often very close to the finite temperature results reported in Pedone et al. [2006]. We were not able to provide a definitive explanation for this observation.

The results for forsterite and orthoenstatite are given in Tables 2 and 3, respectively. In the case of forsterite, while the overall agreement with experiment is relatively good both for the Pedone potential and the potential fit obtained here, the latter produces better results for the overall cell volume $\Omega$ and the elastic constants $C_{11}, C_{22}$, and $C_{33}$. In the case of enstatite, the potential fit predicts even better values of the cell volume $\Omega$, the elastic constants $C_{22}, C_{33}$, and the bulk modulus $B$ (Table 3 ). In the case of fayalite (Table 4), the potential fit here does not demonstrate any real improvement over the Pedone potential. For orthoferrosilite (Table 5), the Pedone potential might be slightly better for the cell volume $\Omega$ and some elastic properties, in particular $C_{11}$, but this is by no means uniform.

Though the dielectric constants of the minerals were not fitted while developing the potential, we examined how well their values were predicted by the newly developed potential. The values were calculated using GULP [Gale and Rohl, 2003] and are tabulated along with the experimental values [Shannon et al., 1991; Olhoeft, 1989] in Table 6. For forsterite and fayalite, we notice that while all three diagonal components of the predicted dielectric constants are much lower than those from experiments, they agreed in their relative strengths with those from experiments. In the case of enstatite and ferrosilite, only an aggregate value of the dielectric constant could be found from experiments. Again, we found that the calculated values were

Table 6. Computed and Experimental Dielectric Constants of Forsterite, Fayalite, Enstatite, and Ferrosilite ${ }^{a}$

\begin{tabular}{|c|c|c|c|c|}
\hline Minerals & Experiment & This Paper & Pedone et al. [2006] & Tilocca et al. [2006] \\
\hline \multirow[t]{3}{*}{ Forsterite } & $\varepsilon_{x x}=7.16$ & $\varepsilon_{x x}=3.53$ & $\varepsilon_{x x}=3.09$ & $\varepsilon_{x x}=6.09$ \\
\hline & $\varepsilon_{y y}=7.61$ & $\varepsilon_{y y}=4.56$ & $\varepsilon_{y y}=3.92$ & $\varepsilon_{y y}=7.34$ \\
\hline & $\varepsilon_{z z}=7.03$ & $\varepsilon_{z z}=3.48$ & $\varepsilon_{z z}=3.11$ & $\varepsilon_{z z}=5.96$ \\
\hline \multirow[t]{3}{*}{ Fayalite } & $\varepsilon_{x x}=8.80$ & $\varepsilon_{x x}=3.89$ & $\varepsilon_{x x}=3.17$ & - \\
\hline & $\varepsilon_{y y}=8.92$ & $\varepsilon_{y y}=5.19$ & $\varepsilon_{y y}=4.12$ & - \\
\hline & $\varepsilon_{z z}=8.58$ & $\varepsilon_{z z}=3.76$ & $\varepsilon_{z z}=3.22$ & - \\
\hline \multirow[t]{3}{*}{ Enstatite } & $\varepsilon=8.4$ & $\varepsilon_{x x}=3.70$ & $\varepsilon_{x x}=3.14$ & $\varepsilon_{x x}=6.08$ \\
\hline & - & $\varepsilon_{y y}=4.11$ & $\varepsilon_{y y}=3.50$ & $\varepsilon_{y y}=6.94$ \\
\hline & - & $\varepsilon_{z z}=4.22$ & $\varepsilon_{z z}=3.17$ & $\varepsilon_{z z}=6.91$ \\
\hline \multirow[t]{3}{*}{ Ferrosilite } & $\varepsilon=8.4$ & $\varepsilon_{x x}=3.78$ & $\varepsilon_{x x}=3.28$ & - \\
\hline & - & $\varepsilon_{y y}=4.28$ & $\varepsilon_{y y}=3.56$ & - \\
\hline & - & $\varepsilon_{z z}=4.33$ & $\varepsilon_{z z}=3.43$ & - \\
\hline
\end{tabular}

${ }^{a}$ Forsterite and fayalite values from experiments are taken from Shannon et al. [1991], and the orthopyroxene values (i.e., enstatite and ferrosilite) are taken from Olhoeft [1989]. The values from Tilocca et al. [2006] were computed using a shell model. 
Table 7. Fractional Coordinates of the Olivine Lattice [Birle et al., $1968]^{a}$

\begin{tabular}{lccc} 
Site & $x$ & $y$ & $z$ \\
\hline $\mathrm{M} 1$ & 0.00000 & 0.00000 & 0.00000 \\
$\mathrm{M} 2$ & 0.98975 & 0.27743 & 0.25000 \\
$\mathrm{M}_{i, 1}$ & 0.52718 & 0.31430 & 0.25000 \\
$\mathrm{M}_{i, 2}$ & 0.08281 & 0.85766 & 0.04677 \\
$\mathrm{Si}$ & 0.42693 & 0.09434 & 0.25000 \\
$\mathrm{O} 1$ & 0.76580 & 0.09186 & 0.25000 \\
$\mathrm{O} 2$ & 0.22012 & 0.44779 & 0.25000 \\
$\mathrm{O} 3$ & 0.27810 & 0.16346 & 0.03431 \\
$\mathrm{O}_{i, 1}$ & 0.52718 & 0.31430 & 0.25000 \\
\hline
\end{tabular}

${ }^{\text {a }}$ Fractional coordinates for the two cation interstitial sites $M_{i, 1}$ and $M_{i, 2}$, and one anion interstitial sites $O_{i, 1}$, determined in the present work, are also given. much lower than the experimental values. The reason for the discrepancy between the experimental values of dielectric constants and those calculated with pair potentials may be due to the fact that in the formalism of pair potentials, dielectric polarization is not taken into account. This conclusion is also supported by our calculation of the dielectric constants with a shell model potential developed by Tilocca et al. [2006]. Using this approach, the calculated values were much closer to experimental values, as shown in Table 6.

To summarize, the potential parameters reported here (Table 1) proved to be a step forward from the original Pedone potential Pedone et al. [2006]. Specifically, the model developed here represents a better fit to the properties of olivine and orthopyroxene minerals. In the results that follow, this improved parameter set was used.

\subsection{Molecular Dynamics Specifics}

In the large-scale molecular dynamics (MD) simulations reported in the next section, we used the Large-scale Atomic/Molecular Massively Parallel Simulator (LAMMPS) simulation code [Plimpton, 1995]. For olivine simulations, the supercell included $11 \times 5 \times 9$ unit cells and a total of 13860 ions. For orthopyroxene, $3 \times 6 \times 10$ unit cells and a total of 14400 ions were simulated. Periodic boundary conditions were applied in all three dimensions. Coulomb interactions were computed using Wolf method using a cutoff of $12 \AA$ and a damping parameter $\alpha=0.2$ [Wolf et al., 1999; Demontis et al., 2001]. The Morse potential was also computed using a cutoff of $12 \AA$. In molecular dynamics simulations, the integration time step was chosen to be $0.5 \mathrm{fs}$ which was found to ensure energy conservation. Moreover, the system was held at constant volume, with the volume corresponding to zero stress in the perfect crystal at finite temperature. Partial charges were used in the MD simulation, with $Q_{\mathrm{Mg}, \mathrm{Fe}}=+1.2 \mathrm{e}, Q_{\mathrm{Si}}=+2.4 \mathrm{e}$, and $Q_{\mathrm{O}}=-1.2 \mathrm{e}$.

It was demonstrated by Aidhy et al. [2009a, 2011] that the kinetics of defect recombination, annihilation, and clustering can be obtained through a focus on the so-called kinetic phase. In other words, defect structures that are observed to evolve after a full simulation of the ballistic phase arising from a high-energy primary knock-on atom might be obtained simply by focusing on the evolution of randomly placed Frenkel pairs. The same approach is taken here. Specifically, we consider an initial distribution of $\mathrm{Mg} / \mathrm{Fe}$ and $\mathrm{O}$ Frenkel defects. In a previous simulation study, it was found that the $\mathrm{SiO}_{2}$ pseudo-Schottky defects were not energetically favorable [Verma and Karki, 2009]. Thus, in the present study, the focus is directed toward understanding Mg/Fe and O Frenkel pairs. However, future investigations could include initial Si Frenkel defects in the initial radiation damaged structure.

\section{Results}

As a first step, stable locations for interstitial $\mathrm{Mg} / \mathrm{Fe}$ cations and $\mathrm{O}$ anions were determined. Candidate interstitial sites were chosen based on local coordination. To determine whether a particular site represents an energetically favorable and stable interstitial site, the system was first relaxed to a ground state and the displacements of the ions from their initial positions were recorded. In exploring possible interstitial sites, a Frenkel defect was placed with the vacancy and interstitial site widely separated so as to avoid any significant interaction. In some cases, candidate interstitial sites were not stable, and instead the system relaxed with a large ion displacement into a structure that corresponded to a different starting interstitial site. At each candidate site, both cation and anion interstitials were considered. Finally, for each stable interstitial site identified, other equivalent sites were generated using the symmetry operations associated with the crystal lattices, in particular using space group Pbnm for olivine, and using space group Pbca for orthopyroxene. 
Table 8. Fractional Coordinates of the Orthopyroxene Lattice [Carlson et al., 1988] ${ }^{\mathrm{a}}$

\begin{tabular}{lccc} 
Site & $\mathrm{x}$ & $\mathrm{y}$ & $\mathrm{z}$ \\
\hline $\mathrm{M} 1$ & 0.3756 & 0.6539 & 0.8684 \\
$\mathrm{M} 2$ & 0.3769 & 0.4846 & 0.3627 \\
$\mathrm{M}_{i, 1}$ & 0.18839 & 0.56192 & 0.14328 \\
$\mathrm{M}_{i, 2}$ & 0.50000 & 0.50000 & 0.50000 \\
$\mathrm{M}_{i, 3}$ & 0.66858 & 0.33379 & 0.80381 \\
$\mathrm{M}_{i, 4}$ & 0.42748 & 0.65659 & 0.56825 \\
$\mathrm{SiA}$ & 0.2715 & 0.3416 & 0.0495 \\
$\mathrm{SiB}$ & 0.4741 & 0.3379 & 0.7967 \\
$\mathrm{O} 1 \mathrm{~A}$ & 0.1834 & 0.3399 & 0.0371 \\
$\mathrm{O} 2 \mathrm{~A}$ & 0.3107 & 0.5019 & 0.0435 \\
$\mathrm{O} 3 \mathrm{~A}$ & 0.3027 & 0.2249 & -0.1714 \\
$\mathrm{O} 1 \mathrm{~B}$ & 0.5627 & 0.3403 & 0.7981 \\
$\mathrm{O} 2 \mathrm{~B}$ & 0.4335 & 0.4844 & 0.6933 \\
$\mathrm{O} 3 \mathrm{~B}$ & 0.4478 & 0.1986 & 0.5988 \\
$\mathrm{O}_{i, 1}$ & 0.1998 & 0.68303 & 0.12735 \\
$\mathrm{O}_{i, 2}$ & 0.50000 & 0.50000 & 0.50000 \\
$\mathrm{O}_{i, 3}$ & 0.55163 & 0.68732 & 0.59068 \\
\hline
\end{tabular}

${ }^{\text {a }}$ Fractional coordinates for the four cation interstitial sites $M_{i, 1}$, $M_{i, 2}, M_{i, 3}$, and $M_{i, 4}$, and three anion interstitial sites $O_{i, 1}, O_{i, 2}$, and $O_{i, 3}$, determined in the present work, are also given.
For olivine, the above procedure identified two stable cation interstitial sites and one stable anion interstitial site. The anion interstitial site turned out to be the same as one of the two cation interstitial sites. For orthopyroxene, four cation interstitial sites and three anion interstitial sites could be identified. One of these interstitial sites was indeed at a center of inversion symmetry, and it was possible to stabilize either a cation or anion there. Along with the stable interstitial sites determined here, we show in Tables 7 and 8 the fractional coordinates of the perfect olivine [Birle et al., 1968] and orthopyroxene [Carlson et al., 1988] crystals, respectively.

Frenkel defects were randomly placed in the crystal lattice [Aidhy et al., 2009a, 2009b, 2011]. Unlike previous

efforts, there was no requirement imposed that the initial interstitial sites and vacancy sites be separated by some minimum distance to prevent recombination in a very short time. Vacant sites were chosen at random with no preference for crystallographically distinct sites. For each system studied, about $1 \%$ of cations and anions were displaced from their perfect crystal sites to generate Frenkel pairs.

After placing the initial Frenkel defects, the structure was relaxed to a zero temperature ground state. During this process, it was found that the many ions in the crystal structure were significantly displaced from their equilibrium lattice site. This could be seen in the snapshots of the defect structure taken before and after the relaxation step (Figure 2). The additional ion displacements occurred due to interactions between defects, which should be fairly long range due to Coulomb interactions and lattice strain. Hereafter, displaced ions are identified as point defects if their coordinates are different from their equilibrium lattice site by more than $1 \AA$. Similarly, a lattice site, cation or anion, is identified as vacant if there is no ion within $1 \AA$ of the equilibrium site. However, not all displaced ions were included to determine the number of Frenkel defects. Specifically, some point defects, while corresponding to significant displacements, result in
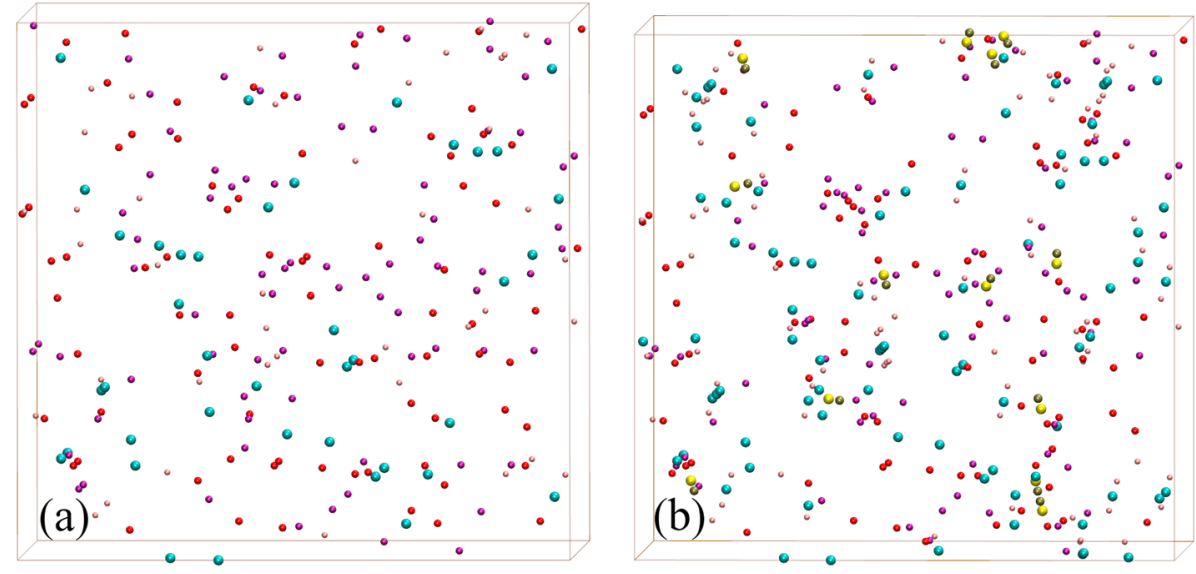

Figure 2. (a) The initial defect structure for olivine (forsterite) is shown before relaxation was carried out in LAMMPS [Plimpton, 1995]. (b) The number of defects has increased due to relaxation and interactions between the defects. Color scheme: Grey spheres = Mg interstitial, blue = Mg vacancy; red = O interstitial, purple = O vacancy; yellow = Si interstitial, green $=$ Si vacancy. 
Table 9. Initial Numbers of Displaced Cations and Cation FPa

\begin{tabular}{lcccc} 
Material & $\begin{array}{c}\text { Mg/Fe FP } \\
\text { (Initial) }\end{array}$ & $\begin{array}{c}\text { Mg/Fe FP } \\
\text { (Final) }\end{array}$ & $\begin{array}{c}\text { Displaced Mg/Fe } \\
\text { (Initial) }\end{array}$ & $\begin{array}{c}\text { Displaced Mg/Fe } \\
\text { (Final) }\end{array}$ \\
\hline Forsterite & 41 & 17 & 81 & 42 \\
Enstatite & 29 & 25 & 34 & 29 \\
Fayalite & 43 & 14 & 103 & 44 \\
Ferrosilite & 28 & 20 & 29 & 27 \\
\hline
\end{tabular}

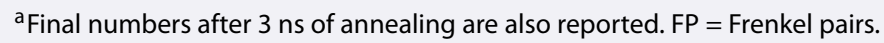

vacancy-interstitial pairs or chains that are not widely separated. Vacancy-interstitial pairs that are separated by a distance less than $1.7 \AA$, which is comparable to the $\mathrm{Si}-\mathrm{O}$ bond length in the perfect crystal, were considered to be part of a chain. In counting the number of Frenkel defects within a chain or cluster of defects, the net excess of interstitials or vacancies in the chain was determined, and that excess was used to determine the effective number of Frenkel defects. One interesting observation was that, although Si Frenkel defects were not included in the starting structure, significant Si displacement occurred during relaxation. This resulted in neighboring $\mathrm{Si}$ interstitial-vacancy pairs, which, by the criterion above, were not considered to be Frenkel defects.

While many of these closely spaced Si vacancy-interstitial pairs exist in the lattice, they do not necessarily annihilate. In fact, it turns out that these local Si displacements are crucial for stabilizing other local defects in the lattice. In particular, we found that the large Si displacements tend to maintain coordination of the $\mathrm{SiO}_{4}$ tetradhedra. As described later, displacements of ions that tend to maintain the coordination of the $\mathrm{SiO}_{4}$ tetrahedra is a mechanism common to both olivine and orthopyroxene, and often involve not only large Si displacements but also large $O$ displacements.

The total number of initial cation and anion Frenkel pairs is shown in Tables 9 and 10. In addition, the number of initial displaced ions in the starting structure is given. The initial defect numbers are obtained after relaxation to the zero temperature ground state. As described above, the number of displaced ions is larger than the number of Frenkel pairs. In Table 9, the number of displaced Si ions in the initial structure is shown. The Si displacements occurred during the initial relaxation, as explained before, but were not counted as Frenkel pairs because the separation between the Si vacancy and Si interstitial was in all cases less than 1.7 ̊. In Table 11, the numbers of the initial Si defects are shown.

The time evolution of the system was computed using MD simulation at constant temperature and volume. The system temperature was maintained at $T=1200 \mathrm{~K}$ for a total time of $3.0 \mathrm{~ns}$. In Figure 3, the number of displaced $\mathrm{Mg} / \mathrm{Fe}$, $\mathrm{Si}$, and $\mathrm{O}$ ions for the olivine minerals forsterite and fayalite are shown as a function of time. The same quantities are shown in Figure 4 for the orthopyroxene minerals enstatite and ferrosilite. Clear differences between the olivine and orthopyroxene minerals can be seen from Figures 3 and 4 . The first clear observation is that the number of displaced ions decreases more rapidly in the olivine minerals in comparison to the orthopyroxene minerals. This is especially evident for displaced $\mathrm{Mg} / \mathrm{Fe}$ cations, which decrease only very gradually with time for orthopyroxene. The number of displaced $\mathrm{O}$ ions decreases substantially with time for each system studied. However, relatively large numbers of displaced $\mathrm{O}$ ions persist in the orthopyroxene minerals in comparison to the olivine minerals. Finally, the number of displaced Si ions changed with time, but the changes were relatively small and were seemingly not systematic.

The time evolution of the total number of displaced ions was found, as expected, to correlate strongly with the fraction of remaining Frenkel pairs in the simulation as a function of time. In Figure 5, the evolution

Table 10. Initial Numbers of Displaced Anions and Anion FPa

\begin{tabular}{lcccc} 
Material & O FP (Initial) & O FP (Final) & Displaced O (Initial) & Displaced O (Final) \\
\hline Forsterite & 64 & 17 & 83 & 31 \\
Enstatite & 57 & 23 & 149 & 66 \\
Fayalite & 64 & 9 & 102 & 18 \\
Ferrosilite & 49 & 20 & 134 & 73 \\
\hline
\end{tabular}

${ }^{\mathrm{a}}$ Final numbers after $3 \mathrm{~ns}$ of annealing are also reported. $\mathrm{FP}=$ Frenkel pairs. 
Table 11. Initial Numbers of Displaced Si and Si FPa

\begin{tabular}{lcccc} 
Material & Si FP (Initial) & Si FP (Final) & Displaced Si (Initial) & Displaced Si (Final) \\
\hline Forsterite & 0 & 0 & 13 & 15 \\
Enstatite & 0 & 0 & 13 & 17 \\
Fayalite & 0 & 0 & 14 & 9 \\
Ferrosilite & 0 & 0 & 18 & 10 \\
\hline
\end{tabular}

${ }^{a}$ Final numbers after 3 ns of annealing are also reported. Note that no Si Frenkel pairs were observed. $\mathrm{FP}=$ Frenkel pairs.

of the number of $\mathrm{Mg}$, Fe, and O Frenkel pairs with simulation time is shown for olivine and orthopyroxene minerals. It is first evident that cation defects heal more slowly than anion defects. Moreover, significant differences are evident between orthopyroxene and olivine. Specifically, cation and anion Frenkel defects heal more slowly in orthopyroxene than in olivine. Later, it will be shown that these differences can be traced to differences in the diffusion behavior and tendencies to trap point defects into larger clusters between the two minerals.

In Figure 6, the mean squared displacements of the ions as a function of time are shown for forsterite and enstatite. In Figure 7, the same quantities are shown for fayalite and ferrosilite. For a very short duration, diffusion can be quite rapid. Over longer times, the rate of diffusion slows due to both vacancy-interstitial annihilation and, as will be seen, trapping of point defects into stable clusters. From Figures 6 and 7, it is evident that cations diffuse more rapidly than anions in olivine. By contrast, in orthopyroxene anion diffusion is more significant than cation diffusion. Rapid oxygen diffusion is especially apparent in enstatite. Finally, while significant Si displacements occur to maintain local coordination of the tetrahedra, the Si ions were not observed to ever exhibit displacements characteristic of diffusion.

The rapid decrease in the number of cation Frenkel defects in olivine is attributed to fast cation diffusion. In contrast to olivine, cation defects diffuse rather slowly in orthopyroxene minerals. As expected, annihilation of Frenkel defects is limited by the diffusion rate. By the same argument, it might be expected that anion Frenkel defects should annihilate more rapidly in orthopyroxene in comparison to olivine due to the observed rapid anion diffusion. However, the data in Figure 5 show otherwise, that is, anion Frenkel defects annihilate more rapidly in olivine. The reasons for this apparent anomaly have to do with strong anisotropy in point defect diffusion observed especially in orthopyroxene.

To demonstrate this point, the $x, y$, and $z$ components of the mean squared displacements are shown in Figure 8 for forsterite and are shown in Figure 9 for enstatite. Here the $x, y$, and $z$ Cartesian directions are chosen along the $a, b$, and $c$ axes, respectively, in the orthorhombic olivine and orthopyroxene unit cells. In Figure 8, cation diffusion shows significant anisotropy, but for anions, anisotropy is less evident. By contrast, in Figure 9 for enstatite, cation diffusion is only weakly anisotropic, whereas anion diffusion is
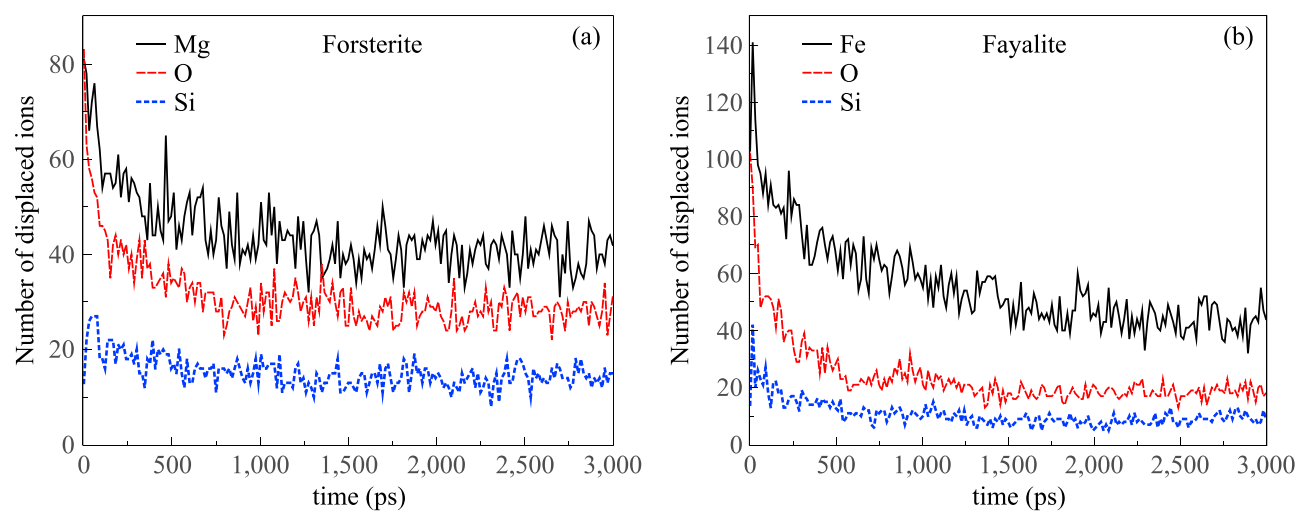

Figure 3. Total number of "displaced ions" (explained in the text) as a function of time for the olivine minerals (a) forsterite and (b) fayalite. 

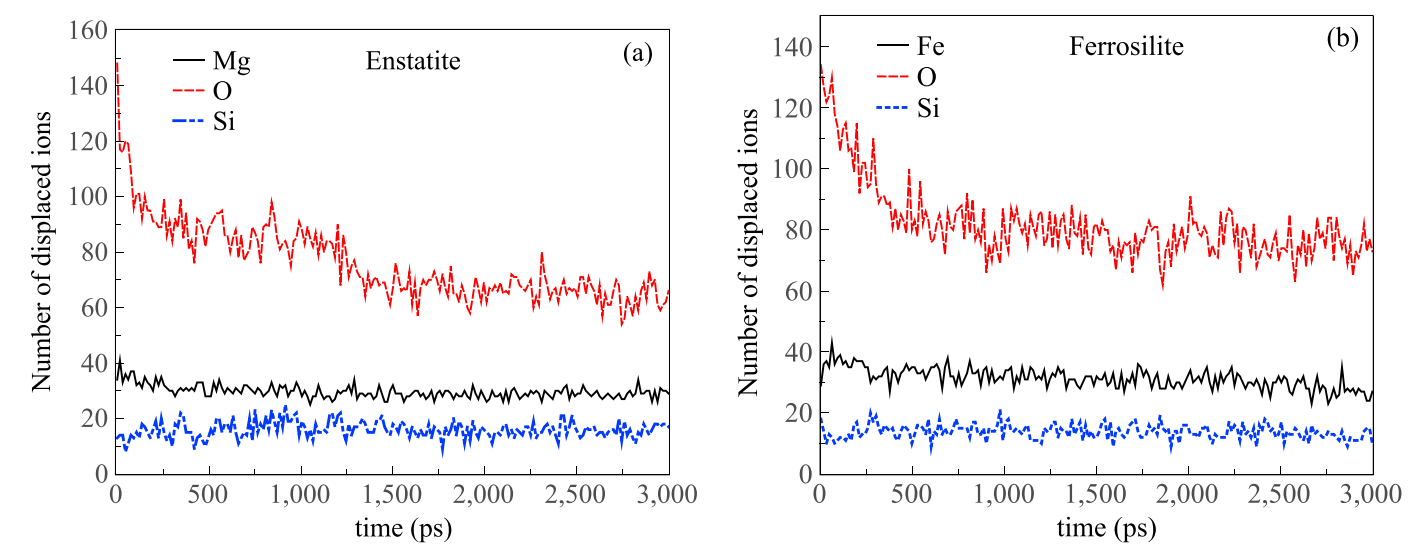

Figure 4. Total number of displaced ions (explained in the text) as a function of time for the orthopyroxene minerals (a) enstatite and (b) orthoferrosilite.

very strongly anisotropic. In particular, anion defects tend to diffuse almost exclusively along the $z$ direction in enstatite, which corresponds to the $c([001])$ crystallographic axis. This explains why anion Frenkel defects heal more slowly in orthopyroxene than in olivine despite the very strong anion diffusion in orthopyroxene. Specifically, because anions diffuse essentially along only one direction, vacancies and interstitials are constrained and hence less likely to annihilate. The most effective mechanism for anion diffusion along the [001] direction in orthopyroxene is found to involve breaking and reforming bonds along the cornerlinked tetrahedra in orthopyroxene. As the oxygen defects migrate along that direction, the system generally maintains the coordination of each $\mathrm{Si}$ ion with the usual four oxygen neighbors. This mechanism, while very effective at transporting oxygen defects, tends to be very inefficient for healing the lattice via interstitial-vacancy annihilation.

Experimental evidence for anisotropic cation diffusion in olivine has been reported by Buening and Buseck [1973] from Mg-Fe interdiffusion experiments. Specifically, it was reported that cation interdiffusion is the highest along the $c$ axis, in agreement with Figure 8. In Lasaga [1980], this effect was explained using atomic-scale defect calculations. Our simulation results for anisotropic diffusion of oxygen are in qualitative agreement with the diffusion experiments reported in Reddy et al. [1980], where it was found that diffusion rates were the largest along the $b$ axis.

Anisotropic cation diffusion had also been observed in enstatite [Schwandt et al., 1998]. Whereas the experiments in Schwandt et al. [1998] suggest that at $T=1200 \mathrm{~K}$, cation diffusion is the highest along the
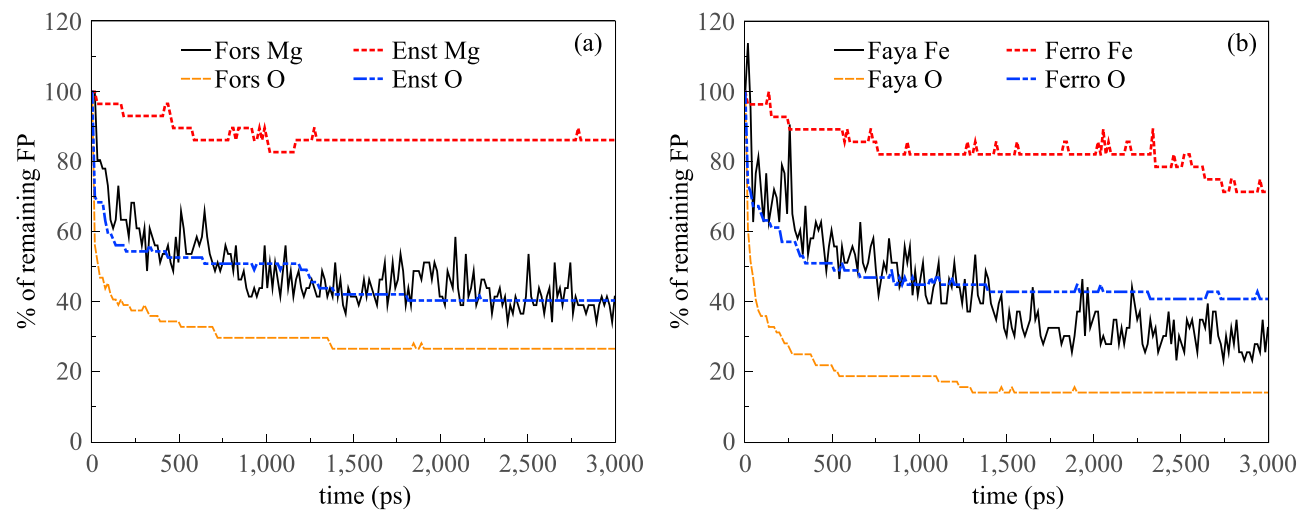

Figure 5. (a) The number of remaining Mg Frenkel pairs (FP) and O FP in forsterite and enstatite is plotted as the percentage of their initial counts versus time. (b) Remaining Fe FP and O FP in fayalite and orthoferrosilite are plotted the same way. The figures clearly indicate that anion FP anneal is much faster in olivine (forsterite and fayalite) than in orthopyroxene (enstatite and ferrosilite). 

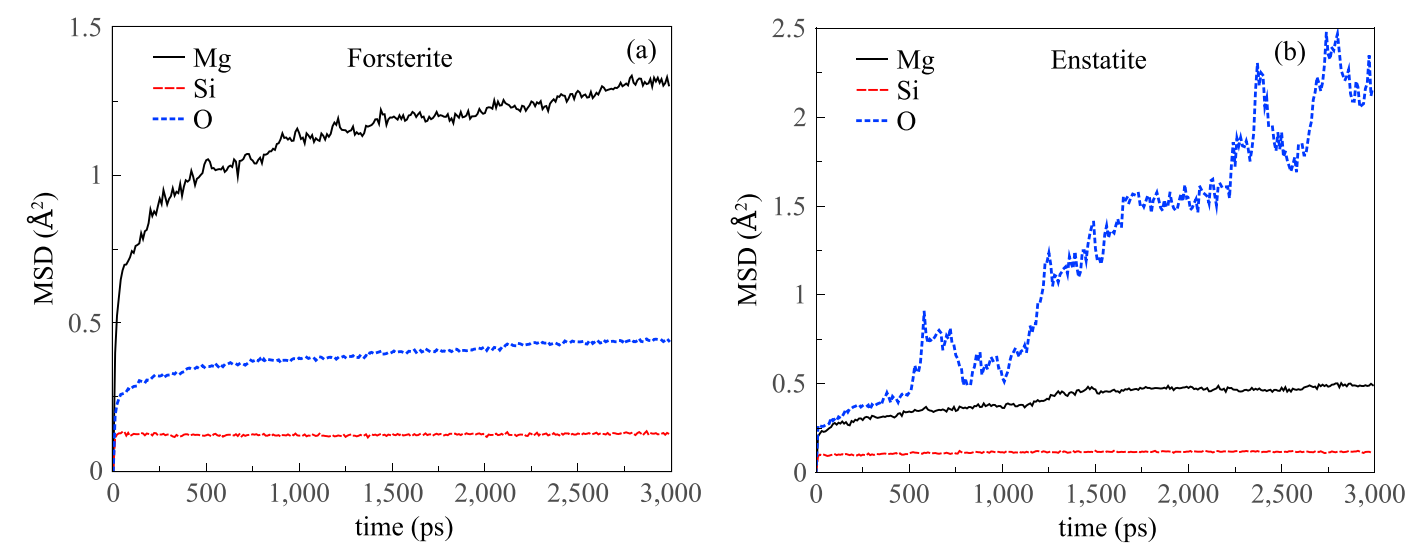

Figure 6. Mean squared displacement of different ion species as a function of time in (a) forsterite and (b) enstatite. While $\mathrm{Mg}$ cation is more mobile in forsterite, $\mathrm{O}$ anion is more mobile in enstatite. Similar difference is also observed in the case of fayalite and ferrosilite (Figure 7), thus indicating that anions are more mobile in olivine than in orthopyroxene.

$c$ axis and the lowest along the $b$ axis, our results (Figure 9) indicate that cation diffusion is the greatest along the $b$ axis. One possible reason for this discrepancy is that the results presented here are dominated by interstitial diffusion, whereas experiments are likely to be dominated by vacancy mediated processes. In fact, in our calculations, we find cation vacancies in enstatite to be immobile within the $3 \mathrm{~ns}$ of simulation time.

The most striking result in Figure 9 is the large and strongly anisotropic oxygen diffusion in enstatite. This result is consistent with the experimental results for diopside [Farver, 1989]. Specifically, it was found by Farver [1989] that oxygen diffusion in diopside along the $c$ axis was 100 times greater than that along the $a$ and $b$ axes. While diopside $\left(\mathrm{CaMgSi}_{2} \mathrm{O}_{6}\right)$ is compositionally and structurally distinct from orthopyroxene, it nevertheless is characterized by an interlocking chains of $\mathrm{SiO}_{4}$ along the $c$ axis. However, experimental results for diopside reported in Farver [1989], Ingrin et al. [2001] show high diffusion rates along both the $a$ and $c$ axes, in contrast to the simulations here for orthopyroxene where only the $c$ axis shows fast anion diffusion.

The decrease in diffusion rate and defect annihilation with time is largely due to trapping of point defects into stable clusters. The dominant mechanism for trapping defects in both olivine and orthopyroxene is to rearrange bonds in the $\mathrm{SiO}_{2}$ network to maintain perfect tetrahedral coordination for the $\mathrm{Si}$ ions. Thus, as bonds break and form during diffusion, $\mathrm{Si}$ ions are most often coordinated with four nearest-neighbor oxygens. In olivine, Coulomb-neutral clusters are quite often found. For example, cation and anion vacancies are often found together in Schottky clusters. To maintain the fourfold coordination of the Si ions when an oxygen vacancy is present, the local structure rearranges so that an oxygen ion is shared between
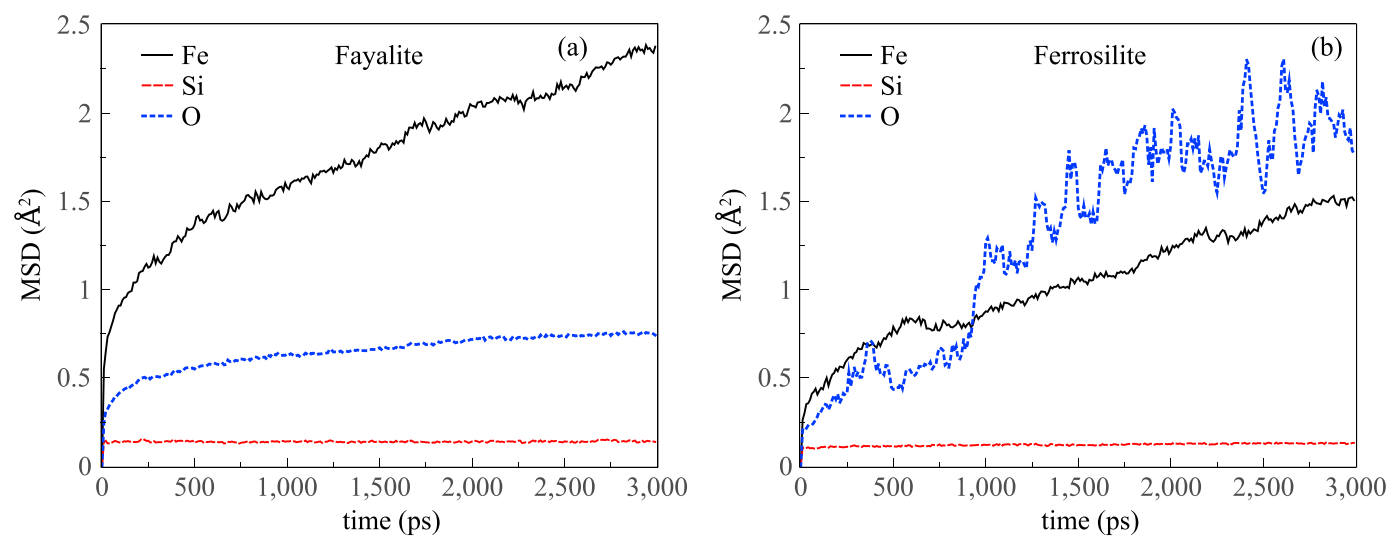

Figure 7. Mean squared displacement of different ion species as a function of time in (a) fayalite and (b) ferrosilite. 

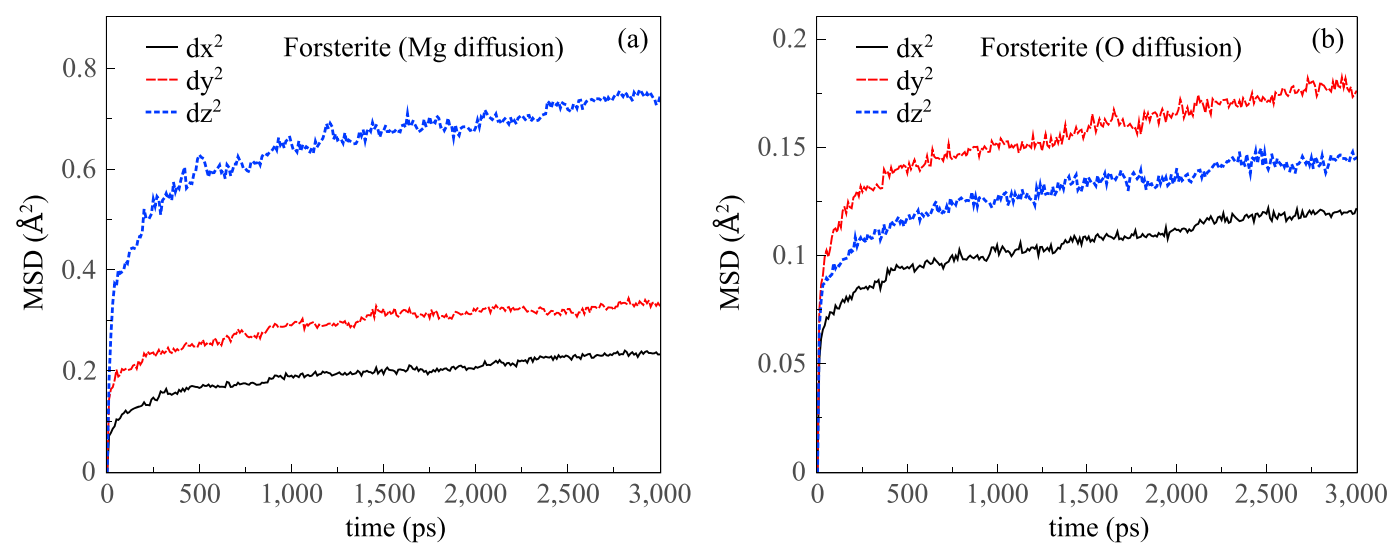

Figure 8. Components of the mean squared displacement of (a) $\mathrm{Mg}$ and (b) $\mathrm{O}$ along the three crystal axes in forsterite. The cation is found to have the highest diffusion rate along $c$ axis, which is in agreement with previous experimental result [Buening and Buseck, 1973].

neighboring tetrahedra. Thus, trapping of cation and anion vacancies in Schottky clusters results in the local olivine structure transforming to short-range order similar to orthopyroxene. In orthopyroxene, where corner sharing between neighboring tetrahedra is intrinsic to the perfect crystal, the perfect coordination of the tetrahedra tends to be preserved by different mechanisms. Specifically, anion vacancies in orthopyroxene can result in local rearrangement of bonds so that additional sharing of oxygen ions occurs between neighboring tetrahedra. Anion interstitials in orthopyroxene tend to result in the breaking of corner-sharing bonds, resulting in independent tetrahedra. Cation interstitials and vacancies tend to result in local rearrangement of the $\mathrm{SiO}_{2}$ network. In contrast to olivine, local trapping of cation and anion defects to make Coulomb-neutral clusters is less effective at trapping defects, probably due to the fact that cation defects are relatively immobile in orthopyroxene even without trapping.

Finally, in orthopyroxene, anion displacements along the $c$ axis, which corresponds to the direction along which tetrahedra are connected by corner-shared bonds, are closely connected to breaking and reforming bonds between neighboring tetrahedra, which results in the very large diffusion rates observed along the $c$ axis.

In olivine, many characteristic clusters that are effective at trapping defects can be identified. In Figure 10, the final defect structures are shown for the olivine minerals forsterite and fayalite. Several $\mathrm{Mg} / \mathrm{Fe}$ split interstitials are found, always with displacement of an ion from an $\mathrm{M} 1$ site. Only a few isolated $\mathrm{Mg} / \mathrm{Fe}$ vacancies are found at M1 sites, consistent with the theoretical results of Lasaga [1980]. For anion defects,
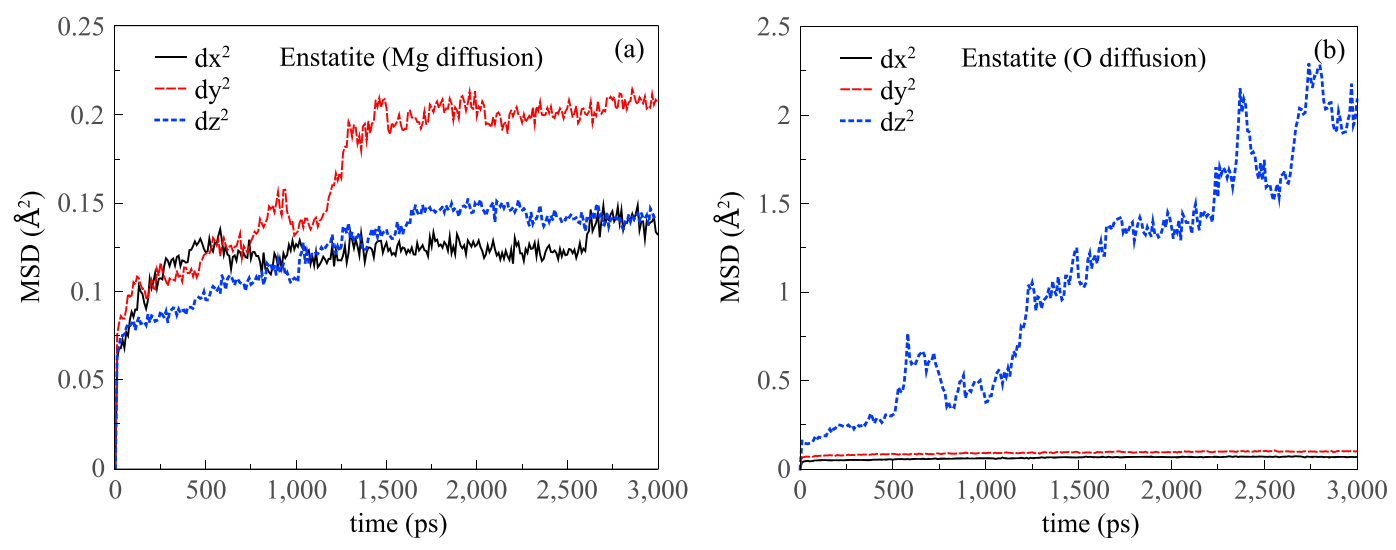

Figure 9. Components of the mean squared displacement of (a) $\mathrm{Mg}$ and (b) $\mathrm{O}$ along the three crystal axes in enstatite. The anion diffusion rate demonstrates large anisotropy, in agreement with experiments [Farver, 1989]. 


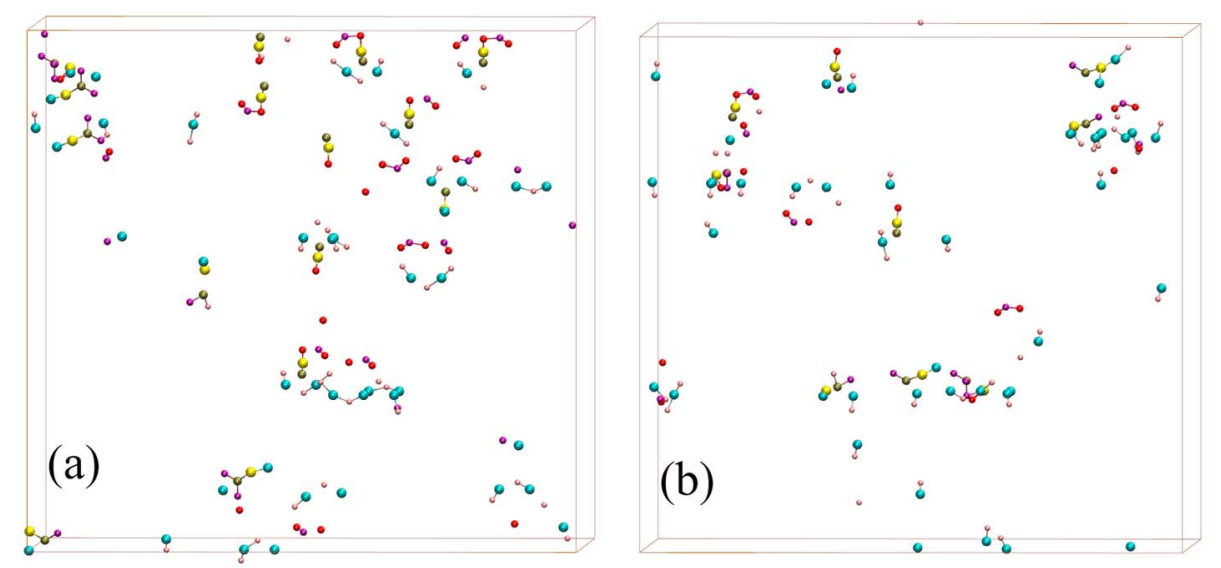

Figure 10. The final defect structures of (a) forsterite and (b) fayalite (annealing time $3 \mathrm{~ns}$ ). The color scheme is the same as used in Figure 2. Comparison of the final defect structure of forsterite with its initial defect structure (Figure $2 \mathrm{~b}$ ) reveals that most of the FP get annihilated, while the surviving FP either form defects cluster or remain as isolated interstitials and vacancies.

many instances of split interstitials were found. By contrast, no cases of split anion vacancies were observed. In previously reported density functional theory (DFT) calculations, split cation interstitials were shown to be energetically favorable over various tetrahedral and octahedral sites [Walker et al., 2009]. In the present calculations, this seems to result in isolated cation interstitials forming split interstitial defects. For split anion interstitials and split cation vacancies, there appear to be no DFT calculations verifying their existence.

Most often, cation and anion vacancies are trapped in clusters in olivine. These include isolated nearest-neighbor Schottky clusters at M1-O2 or M1-O3 sites. In addition, vacancies at M2 and O3 nearest-neighbor sites lead to a bound pair with formation of a split cation vacancy. There are also many examples of bound Schottky clusters at second nearest-neighbor sites. For example, we found bound pairs at second nearest-neighbor M2 and 01 sites. One common structure that was observed particularly in forsterite consists of two cation vacancies and two anion vacancies occurring at second nearest-neighbor

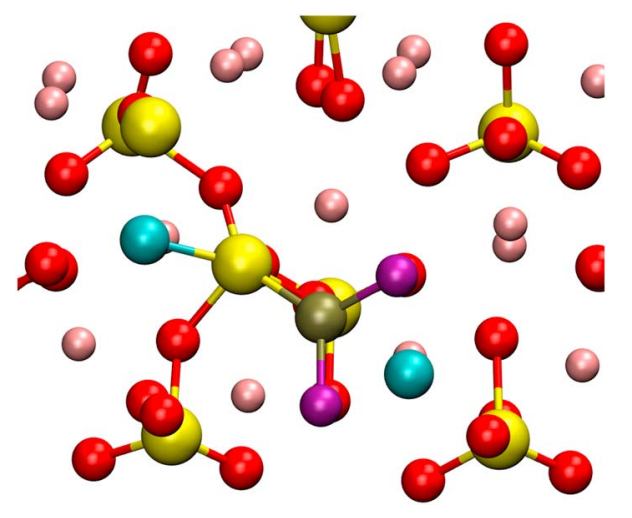

Figure 11. Structure of a common defect cluster observed in olivine. The structure consists of a displaced $\mathrm{Si}$, two oxygen vacancies (purple spheres), and two $\mathrm{Mg}$ vacancies (blue spheres). One of the $\mathrm{Mg}$ vacancies is nearest neighbor to both the oxygen vacancies, while the other is located at a second nearest-neighbor position. The Si inside the tetrahedron which contains the oxygen vacancies undergoes a significant displacement, thereby trapping two MgO Schottky pairs and forming a charge neutral cluster. The displaced Si shares new bonds with neighboring tetrahedra to maintain its fourfold coordination. sites. These clusters also involve large displacement of a Si ion to maintain the perfect fourfold coordination of the Si. In particular, a Si with two neighboring anion vacancies is displaced to form two corner-sharing bonds with neighboring tetrahedra. In Figure 11, a defect cluster of this type observed in forsterite is shown. Interestingly, we found that often Schottky clusters formed at second nearest-neighbor sites were quite stable and tend to prevent the formation of nearest-neighbor bound Schottky clusters.

The defect clusters described above are consistent with a local transformation from olivine to pyroxene. In olivine minerals, this represents the most important trapping mechanism for point defects that inhibits vacancy-interstitial recombination. Transformation of olivine to an amorphous material with pyroxene composition is thought to occur due to ion irradiation of interplanetary dust particles [Rietmeijer, 2009]. However, in that case, the transformations are due to an overall change in composition due to radiation damage. By contrast, the MD simulations describe local transformations that occur while the overall composition is stoichiometric to olivine. 


\begin{tabular}{|c|c|c|c|c|}
\hline Schottky Pair & $\begin{array}{l}\text { Nearest-Neighbor } \\
\text { Energy }\end{array}$ & $\begin{array}{c}\text { Second Nearest-Neighbor } \\
\text { Energy }\end{array}$ & $\begin{array}{l}\text { Widely Separated } \\
\text { Scottky Pair Energy }\end{array}$ & $\begin{array}{l}\text { Calculated From } \\
\text { Walker et al. [2009] }\end{array}$ \\
\hline M1O1 & 4.66 & 4.15 & 6.12 & 11.14 \\
\hline $\mathrm{M} 1 \mathrm{O} 2$ & 2.99 & - & 4.27 & 8.37 \\
\hline $\mathrm{M} 1 \mathrm{O} 3$ & 3.38 & - & 4.67 & 7.71 \\
\hline M2O1 & 5.96 & 3.99 & 7.27 & 13.06 \\
\hline $\mathrm{M} 2 \mathrm{O} 2$ & 4.03 & 4.58 & 5.42 & 10.29 \\
\hline $\mathrm{M} 2 \mathrm{O} 3$ & 4.24 & 3.35 & 5.82 & 9.63 \\
\hline
\end{tabular}

avalues are given in $\mathrm{eV}$.

Some of the general trends of defect clustering were elucidated by computing the energies of isolated Schottky clusters. The formation energies are determined with respect to perfect crystal MgO, or FeO. Specifically, the energy of an MgO Schottky defect in forsterite was determined by computing the energy of supercells of perfect crystal forsterite with $N \mathrm{MgO}$ units, defective crystal forsterite with a single Schottky defect and $(N-1) \mathrm{MgO}$ units, and perfect crystal MgO. The energy $E_{S}$ for a Schottky defect was computed using

$$
E_{S}=E_{(N-1)}^{F o}+E_{\mathrm{MgO}}-E_{N}^{F o}
$$

where $E_{(N-1)}^{F o}$ is the energy of a supercell of defective forsterite with a single Schottky defect, $E_{N}^{F o}$ is the energy of a supercell of perfect crystal forsterite, and $E_{\mathrm{MgO}}$ is the energy of a single formula unit of $\mathrm{MgO}$ in the rock salt structure. The size of the supercell used for the forsterite calculations was the same as those used in the defect annealing, so that the number of MgO units was $N=3960$ in the perfect crystal and $(N-1)=3959$ in the defective cell. Computed in this way, the energy is defined as the energy per MgO Schottky defect. Because the calculations were performed with relatively large simulation cells with dimension along any direction $\sim 50 \AA$, interactions that occur due to periodic boundary conditions should be small, which justifies the use of supercell methods rather than the Mott-Littleton approach. Moreover, the defect energies computed for the supercell should be most relevant to the energetics of defects in the annealed system. In Table 12, we show computed formation energies for nearest-neighbor, second nearest-neighbor, and widely separated Schottky pairs. The relatively low formation energies for M1-O2, M1-O3, and M2-O3 Schottky clusters are consistent with their observed presence in the annealed structures. The other possible nearest-neighbor pairs all result in significantly higher formation energies and, hence, were not observed in the annealed structures. Note also that relaxation of the M2-O3 cluster resulted in the formation of a split cation vacancy bound to the anion vacancy, as was found in the annealed structures. Table 12 also shows the energy of Schottky defects where the two vacancies were widely separated but remained in the same supercell. As expected, these energies are larger than those of the Schottky clusters, since the Schottky clusters are bound attractively by Coulomb interactions. For a comparison, the energy values from Walker et al. [2009] are also tabulated, and they turn out to be substantially larger than our calculated values. The reasons for this disagreement are twofold: first, the values reported in Walker et al. [2009] are computed using the Mott-Littleton method as implemented in GULP [Gale and Rohl, 2003] and hence do not include the attractive interaction between the vacancies in the Schottky pair. These values, therefore, most closely correspond to the values we obtained with widely separated Schottky pairs. Second, Walker et al. [2009] uses an empirical potential with formal point charges [Walker et al., 2003], whereas we have used partial charges. The use of formal point charges is likely to exaggerate the ionic nature of olivine and thereby magnify the Schottky energies. Thus, due to differences in computational models and methods, a quantitative comparison cannot be made between our results and those reported in Walker et al. [2009]. Nonetheless, Table 12 demonstrates similar trends in both sets of Schottky energies. It is clear that in both cases, the M2-O1 nearest-neighbor Schottky pair results in the largest Schottky defect energy. Both the $\mathrm{O} 2$ and $\mathrm{O} 3$ sites are more favorable than the $\mathrm{O} 1$ sites for oxygen vacancies. Also, M1 sites are significantly more favorable for $\mathrm{Mg}$ vacancies than the M2 sites.

A certain type of extended defect cluster, which is seen in the final forsterite and fayalite structures, involves an $\mathrm{MgO}$ (FeO) Schottky pair with a displaced $\mathrm{Si}$ ion. In this cluster, the $\mathrm{Si}$ is displaced by $\sim 1.6 \AA$ toward the $\mathrm{Mg}(\mathrm{Fe})$ vacancy. Near the original Si site, an O vacancy was generally observed. The sites for the Mg (Fe) 
Table 13. Frenkel Defect Energies for Forsterite ${ }^{a}$

\begin{tabular}{lcc} 
Frenkel Pair & This Work & $\begin{array}{c}\text { Calculated From } \\
\text { Walker et al. [2009] }\end{array}$ \\
\hline $\mathrm{Mg}_{i, 1}$ & 4.35 & - \\
$\mathrm{Mg}_{i, 2}$ & 4.34 & - \\
Split Mg interstitial & 4.88 & 6.73 \\
$\mathrm{O}_{i, 1}$ & 6.27 & 9.09 \\
\hline
\end{tabular}

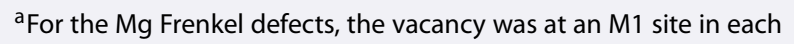
case. For the oxygen Frenkel defect, the vacancy was at an $\mathrm{O} 2$ site. Values are given in eV. and $O$ vacancies were found to be second nearest-neighbor sites. The vacant sites for these extended clusters were found in M1-O1, M2-O1, $\mathrm{M} 2-\mathrm{O} 2$, and $\mathrm{M} 2-\mathrm{O} 3$ combinations. The formation energies of these second nearest-neighbor Schottky pairs are also shown in Table 12. It can be seen that in some cases, the energy of the extended, second nearest-neighbor cluster is lower than the formation energy of corre-

sponding nearest-neighbor Schottky pair. This suggests that Schottky clusters are readily trapped at second nearest-neighbor sites, thereby preventing the formation of the first nearest-neighbor Schottky clusters.

The formation energy of the Schottky cluster shown in Figure 11 was also computed. As mentioned before, clusters of this type were frequently found in forsterite. However, no such cluster was observed in the annealed fayalite. The energy of formation of this entire cluster was calculated to be $4.194 \mathrm{eV}$. Since the structure involved two MgO Schottky pairs, this means formation energy of each Schottky pair was $2.097 \mathrm{eV}$, which is significantly lower than the lowest formation energy among all the nearest-neighbor MgO Schottky pairs shown in Table 12. This explains the stability of these clusters and their frequency in the annealed forsterite structure.

The energies of various Frenkel defects in forsterite were also computed and compared with those reported in Walker et al. [2009] in Table 13. The energy of a Frenkel defect was determined by placing an interstitial and a vacancy at distant sites. In the present work, in contrast to Walker et al. [2009] where only split interstitials were observed, we found that it was possible to stabilize $\mathrm{Mg}$ interstitials at two distinct sites $M_{i, 1}$ and $\mathrm{M}_{i, 2}$. However, in the annealed structure, split $\mathrm{Mg}$ interstitials with the same structure as that reported in Walker et al. [2009] were indeed observed, and the energy of this structure is also given in Table 13. The values computed here are somewhat lower than those reported in Walker et al. [2009], apparently due to the fact that partial charges were used in our work. Interestingly, in our calculations we find that Frenkel defects that correspond to a split Mg interstitial are somewhat higher in energy than the Frenkel defects associated with the two distinct $M_{i}$ sites. We did not explore the energies of near vacancy-interstitials pairs, which would be expected to be bound by Coulomb interactions in the same way that Schottky clusters are bound.

The evolution of orthopyroxene minerals with Frenkel defects exhibits not only some mechanisms in common with olivine but also some unique characteristics. In Figure 12, the point defect structure of
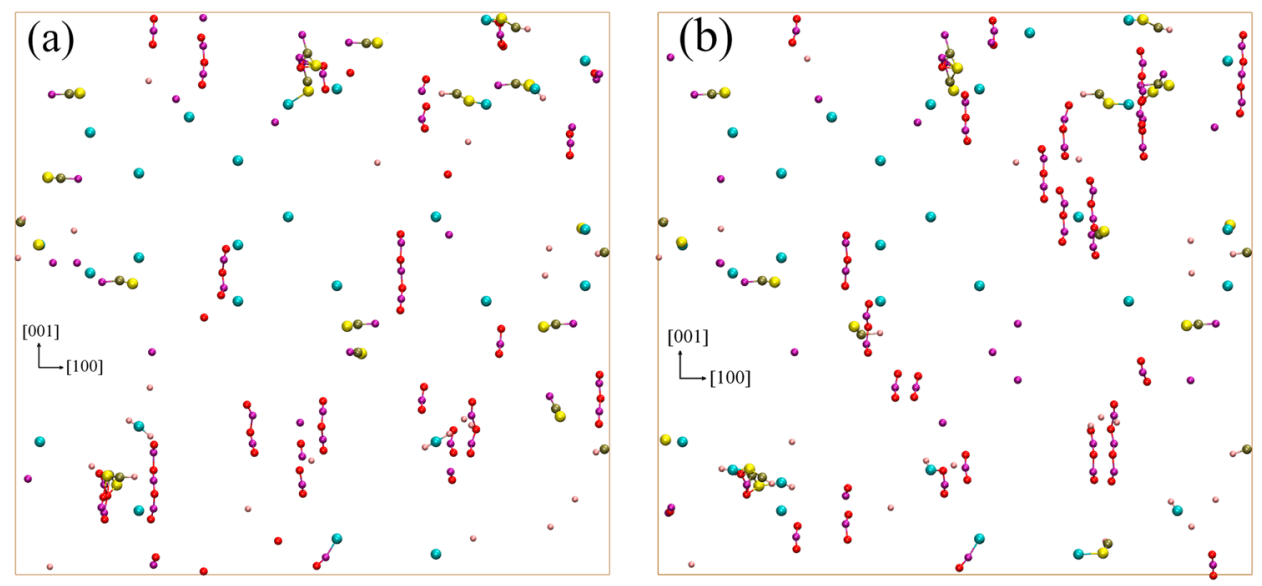

Figure 12. Snapshots of enstatite defect structures at (a) $2.5 \mathrm{~ns}$ and (b) $3 \mathrm{~ns}$. A close examination of these figures reveals that while the Mg interstitials (grey spheres) and Mg vacancies (blue spheres) remain essentially trapped and immobile, long-connected chains of oxygen vacancies (purple spheres) and oxygen interstitials (red spheres) continuously appear and disappear (annihilate) along the [001] direction. 

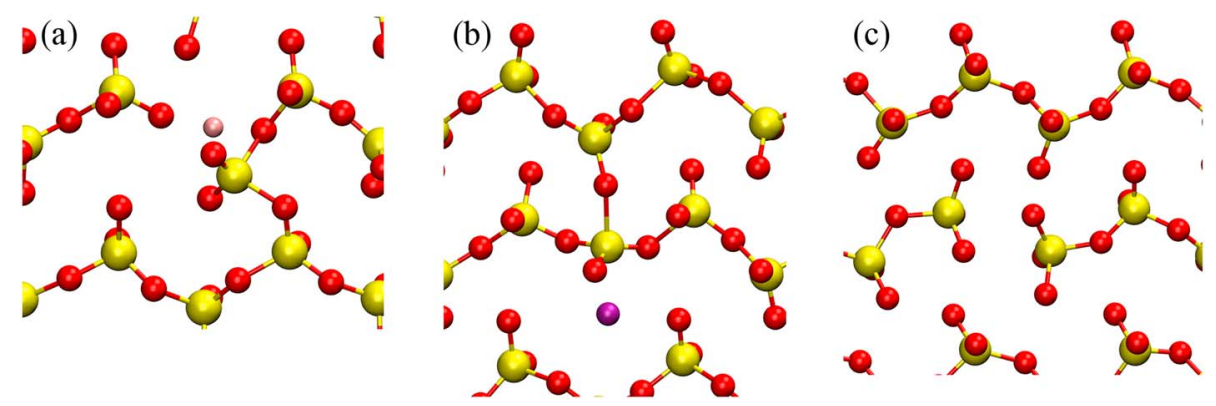

Figure 13. Different mechanisms in orthopyroxene that occur in response to local defects which act to maintain the fourfold coordination of the Si ions, as described in the text. The $c$ axis is along the horizontal. (a) A Mg interstitial (grey sphere) causes rearrangement of the $\mathrm{SiO}_{2}$ network. (b) An oxygen vacancy (purple sphere) results in an additional bond joining neighboring tetrahedra. (c) An extra oxygen interstitial results in the chain of tetrahedra becoming broken.

orthopyroxene is shown after 3 ns of simulation time for ferrosilite. From this figure, it is evident that the dominant mechanism of evolution is the migration of anion interstitials along the $c$ axis. This results in chains of anion vacancies and interstitials. A closer look at these chains reveals that there is only one extra anion interstitial in each chain. This is consistent with the large, anisotropic diffusion of anion described previously. In contrast to the behavior of anion interstitials, cation defects and isolated anion vacancies are fairly immobile. This is consistent with the observation of slow healing rates in orthopyroxene. Specifically, while anion interstitials are extremely mobile, diffusion only occurs along the $c$ axis. Hence, the likelihood of an anion interstitial encountering an anion vacancy for recombination is relatively low. Moreover, the slow diffusion rates for cation defects and anion vacancies also make trapping into extended clusters less likely to occur.

As with olivine, the presence of isolated cation or anion defects in orthopyroxene can result in significant displacements of $\mathrm{Si}$ ions. These displacements take place as the Si tetrahedra try to restore their fourfold coordination through reorganization of the $\mathrm{SiO}_{2}$ network. In particular, displacements of the $\mathrm{Si}$ ions tend to break the bonds that join tetrahedra along the $c$ axis and alternately form new bonds in the perpendicular directions. Hence, Si displacements primarily occur perpendicular to the $c$ axis. It is also apparent that large Si displacements tend to act in a way that immobilizes cation defects and prevents annihilation of cation Frenkel defects. Specifically, there are several instances in orthopyroxene where Si ions are displaced away from a cation interstitial toward a cation vacancy. In this structure, the cation interstitial tends to relax toward the vacancy Si site. This essentially results in the Frenkel pair being stabilized by the formation of (Mg and Fe)/Si antisite defects.

As mentioned above, displacements of ions and migration of defects tend to occur to maintain the fourfold coordination of the tetrahedra. Some of these mechanisms are shown in Figure 13, in this case for enstatite. In Figure 13a, a Mg interstitial and an O interstitial are bound together, resulting in a disruption of the bonds joining neighboring tetrahedra. In Figure 13b, the presence of an isolated $O$ vacancy is stabilized by a bond joining tetrahedra along a direction perpendicular to the $c$ axis. Finally, in Figure $13 c$, an $\mathrm{O}$ interstitial results in disruption of bonds joining neighboring tetrahedra together. In short, defects are accommodated in the lattice by rearrangement of the $\mathrm{SiO}_{2}$ network, with bonds breaking and forming so that each $\mathrm{Si}$ ion tends to be coordinated with four $\mathrm{O}$ ions.

\section{Conclusions}

Experiments reported in Wang et al. [1998] indicate that olivine is more resistant than orthopyroxene to amorphization due to radiation damage. The same trend was reported in Christoffersen and Keller [2011], although the results were accurate within the experimental error. The results presented here suggest an explanation for these observations. Specifically, cation and anion Frenkel defects were found in the simulated annealing to heal more rapidly in olivine due to strong differences in the diffusion pattern in the two crystal structures. In orthopyroxene, oxygen defects migrate rapidly along the $c$ axis by a mechanism which rearranges the bonds between $\mathrm{SiO}_{4}$ tetrahedra. This phenomena appears to lead to local disruption of the oxygen sublattice and potential amorphization of the tetrahedral network. Although the oxygen dif- 
fusion in orthopyroxene is rapid, its one-dimensional nature renders it very inefficient at healing Frenkel defects. In olivine, by contrast, diffusion was found to be more isotropic. Consequently, even though the defects in olivine can become easily trapped in clusters, there is also a greater probability of annihilation of Frenkel defects and healing of the crystalline lattice. In short, we predict that significant differences in the mechanisms and the rates of defect diffusion between olivine and orthopyroxene are responsible for observed differences in amorphization thresholds. Rapid oxygen diffusion in orthopyroxene, with its tendency to disorder the $\mathrm{SiO}_{2}$ network, is the most dramatic difference between the two crystal structures.

While most closely related to radiation damage experiments, the results here may also shed some light on the pulsed laser experiments reported in Yamada et al. [1999], Sasaki et al. [2001], and Sasaki and Kurahashi [2004], where changes in the optical reflectivity in olivine were found to be more significant than that in orthopyroxene. Changes in the optical reflectivity occur mainly due to reduction of the silicate surface, generally accompanied by the formation of elemental Fe clusters. This has led to the suggestion that weathering rates of olivine in the space environment are greater than the rates in orthopyroxene. Given the apparent tendency of olivine to be more resistant to radiation damage, this might at first seem surprising. The key to understand this is to recognize that the laser heating experiments were primarily focused on optical effects and the production of $\mathrm{npFe}^{0}$, whereas TEM analysis of radiation damage was focused on determining the critical dose for amorphization, and not necessarily on reduction. We propose that as the surface is reduced, oxygen should diffuse from the bulk of the system to replenish the oxygen lost at the surface. The simulations reported here show that orthopyroxene is significantly more efficient at transporting oxygen from the bulk to the surface, thereby inhibiting strong reduction of the surface. By contrast, the larger cation mobility in olivine should tend to result in the rapid formation and growth of Fe inclusions that are characteristic of space weathering.

These predictions suggest specific features that might be observed in strongly reduced materials, either produced in natural space weathering or in the laboratory via pulsed laser heating. In particular, if oxygen lost on the surface is replenished by diffusion from deeper in the bulk, then surface reduction should be less significant on orthopyroxene, but might penetrate deeper into the bulk. For olivine, oxygen is less mobile, and hence surface reduction might be expected to be more significant at the surface, but with more shallow penetration into the bulk. Surface reduction of olivine should also be stabilized by the more rapid cation diffusion and formation of Fe metal inclusions.

While calculations of the kind presented here might be useful in providing insights into radiation damage phenomena in silicate minerals, it is also clear that there are several limitations, the most important one being that they assume fixed point charges and stoichiometric minerals. In atomic-scale study with pair-potentials, point defects can only lead to local deviations from stoichiometry, while maintaining overall stoichiometry of either olivine or orthopyroxene. Thus, reduction of the mineral surface, and the gradual formation of metallic Fe clusters, cannot be directly explored by the methodology. To go beyond this level of calculation, we are developing a Charge-Optimized Many-Body potential [Cheng et al., 2014, and references therein] for the Fe-Si-O system. This approach uses variable point charges and allows for the formation of elemental metal and deviations from perfect stoichiometry. Hence, the evolution of the olivine and orthopyroxene systems can be determined as oxygen is lost due to heating or preferential sputtering due to radiation damage. Work along these lines can be expected to address the question of the relative roles of vapor deposition and radiation damage (e.g., via preferential sputtering of oxygen) in the production of $\mathrm{nFFe}^{0}$, which would be especially relevant in understanding apparent differences between what we understand about lunar samples and more recent observations from Itokawa.

Acknowledgments
This work was supported by a grant from the Florida Space Institute. The authors also acknowledge the University of Central Florida Stokes Advanced Research Computing Center for providing computational resources and support that have contributed to results reported herein (http:// webstokes.ist.ucf.edu). The data generated in this work, specifically the input files used with LAMMPS and GULP simulation codes and the output files, can be obtained by e-mail to patrick.schelling@ucf.edu.

\section{References}

Aidhy, D. S., P. C. Millett, D. Wolf, S. R. Phillpot, and H. Huang (2009a), Kinetically driven point-defect clustering in irradiated MgO by molecular-dynamics simulation, Scr. Mater., 60, 691-694, doi:10.1016/j.scriptamat.2008.12.052.

Aidhy, D. S., P. C. Millett, T. Desai, D. Wolf, and S. R. Phillpot (2009b), Kinetically evolving irradiation-induced point defect clusters in UO 2 by molecular dynamics simulation, Phys. Rev. B: Condens. Matter, 80, 104107, doi:10.1103/PhysRevB.80.104107.

Aidhy, D. S., D. Wolf, and A. El-Azab (2011), Comparison of point-defect clustering in irradiated $\mathrm{CeO}_{2}$ and $\mathrm{UO}_{2}$ : A unified view from molecular dynamics simulations and experiments, Scr. Mater., 65, 867-870, doi:10.1016/j.scriptamat.2011.07.051.

Bass, J. D. (1995), Elasticity of minerals, glasses, and melts, in Mineral Physics and Crystallography: A Handbook of Physical Constants, edited by T. J. Ahrens, pp. 45-63, AGU, Washington, D. C., doi:10.1029/RF002.

Birle, J. D., G. V. Gibbs, P. B. Moore, and J. V. Smith (1968), Crystal structures of natural olivines, Am. Mineral., 53, $807-824$. Buening, D. K., and P. R. Buseck (1973), Fe-Mg lattice diffusion in olivine, J. Geophys. Res., 78, 6852-6862, doi:10.1029/JB078i029p06852. 
Carlson, W. D., J. S. Swinnea, and D. E. Miser (1988), Stability of orthoenstatite at high temperature and low pressure, Am. Mineral., 73, $1255-1263$.

Carrez, P., K. Demyk, P. Cordier, L. Gengembre, J. Grimblot, L. D'Hendecourt, A. P. Jones, and H. Leroux (2002), Low-energy helium ion irradiation-induced amorphization and chemical changes in olivine: Insights for silicate dust evolution in the interstellar medium, Meteorit. Planet. Sci., 37, 1599-1614, doi:10.1111/j.1945-5100.2002.tb00814.x.

Cassidy, W., and B. Hapke (1975), Effects of darkening processes on surfaces of airless bodies, Icarus, 25, 371-383, doi:10.1016/0019-1035(75)90002-0.

Chapman, C. R. (2004), Space weathering of asteroid surfaces, Annu. Rev. Earth Planet. Sci., 32, 539-567, doi:10.1146/annurev.earth.32.101802.120453.

Cheng, Y.-T., T. Liang, J. A. Martinez, S. R. Phillpot, and S. B. Sinnott (2014), A charge optimized many-body potential for titanium nitride (TiN), J. Phys. Condens. Matter, 26, 265004, doi:10.1088/0953-8984/26/26/265004.

Christoffersen, R., and L. P. Keller (2011), Space radiation processing of sulfides and silicates in primitive solar systems materials: Comparative insights from in situ TEM ion irradiation experiments, Meteorit. Planet. Sci., 46(7), 950-969, doi:10.1111/j.1945-5100.2011.01203.x.

Christoffersen, R., D. S. McKay, and L. P. Keller (1996), Microstructure, chemistry, and origin of grain rims on ilmenite from the lunar soil finest fraction, Meteorit. Planet. Sci., 31, 835-848, doi:10.1111/j.1945-5100.1996.tb02117.x.

Demontis, P., S. Spanu, and G. B. Suffritti (2001), Application of the Wolf method for the evaluation of Coulombic interactions to complex condensed matter systems: Aluminosilicates and water, J. Chem. Phys., 114, 7980-7988, doi:10.1063/1.1364638.

Farver, J. R. (1989), Oxygen self-diffusion in diopside with application to cooling rate determinations, Earth Planet. Sci. Lett., 92, 386-396, doi:10.1016/0012-821X(89)90062-9.

Gale, J. D., and A. L. Rohl (2003), The General Utility Lattice Program (GULP), Mol. Simul., 29, 291-341, doi:10.1080/0892702031000104887.

Golubov, S. I. (2012), Radiation damage theory, in Comprehensive Nuclear Materials, vol. 1, edited by R. J. M. Konings et al., pp. 357-391, Elsevier, Amsterdam, doi:10.1016/B978-0-08-056033-5.00029-X.

Hapke, B. (2001), Space weathering from Mercury to the asteroid belt, J. Geophys. Res., 106, 10,039-10,073, doi:10.1029/2000JE001338.

Hazen, R. M. (1976), Effects of temperature and pressure on the crystal structure of forsterite, Am. Mineral., 61, 1280-1293.

Ingrin, J., L. Pacaud, and O. Jaoul (2001), Anisotropy of oxygen diffusion in diopside, Earth Planet. Sci. Lett., 192, 347-361, doi:10.1016/S0012-821X(01)00460-5.

Keller, L. P., and D. S. McKay (1993), Discovery of vapor deposits in the lunar regolith, Science, 261, 1305-1307, doi:10.1126/science.261.5126.1305.

Keller, L. P., and D. S. McKay (1997), The nature and origin of rims on lunar soil grains, Geochim. Cosmochim. Acta, 61, 2331-2341, doi:10.1016/S0016-7037(97)00085-9.

Kubota, A., M.-J. Caturla, J. Stolken, B. Sadigh, S. Reyes, T. D. de la Rubia, and J. F. Latkowski (2003), Threats to ICF reactor materials: Computational simulations of radiation damage induced topological changes in fused silica, Nucl. Instrum. Methods Phys. Res., Sect. B, 202, 88-92, doi:10.1016/S0168-583X(02)01837-2.

Lasaga, A. C. (1980), Defect calculations in silicates: Olivine, Am. Mineral., 65, 1237-1248.

Noguchi, T., et al. (2014), Space weathered rims found on the surfaces of the Itokawa dust particles, Meteorit. Planet. Sci., 49, 188-214, doi:10.1111/maps.12111.

Olhoeft, G. R. (1989), Electrical properties of rocks, in CINDAS Data Series on Material Properties, Volume Il-2, Physical Properties of Rocks and Minerals, edited by Y. S. Touloukian, W. R. Judd, and R. F. Roy, pp. 257-329, Hemisphere, New York.

Pedone, A., G. Malavasi, M. C. Menziani, A. N. Cormack, and U. Segre (2006), A new self-consistent empirical interatomic potential model for oxides, silicates, and silica-based glasses, J. Phys. Chem. B, 110, 11,780-11,795, doi:10.1021/jp0611018.

Plimpton, S. (1995), Fast parallel algorithms for short-range molecular dynamics, J. Comput. Phys., 117, 1-19, doi:10.1006/jcph.1995.1039.

Reddy, K. P. R., S. M. Oh, L. D. Major, and A. R. Cooper (1980), Oxygen diffusion in forsterite, J. Geophys. Res., 85, 322-326, doi:10.1029/JB085iB01p00322.

Rietmeijer, F. J. M. (2009), The irradiation-induced olivine to amorphous pyroxene transformation preserved in an interplanetary dust particle, Astrophys. J., 705, 791-797, doi:10.1088/0004-637X/705/1/791.

Sasaki, S., and E. Kurahashi (2004), Space weathering on Mercury, Adv. Space Res., 33, 2152-2155, doi:10.1016/S0273-1177(03)00442-3.

Sasaki, S., K. Nakamura, Y. Hamabe, E. Kurahashi, and T. Hiroi (2001), Production of iron nanoparticles by laser irradiation in a simulation of lunar-like space weathering, Nature, 410, 555-557, doi:10.1038/35069013.

Schwandt, C. S., R. T. Cygan, and H. R. Westrich (1998), Magnesium self-diffusion in orthoenstatite, Contrib. Mineral. Petrol., 130, 390-396, doi:10.1007/s004100050373.

Shannon, R., M. Subramanian, S. Hosoya, and G. Rossman (1991), Dielectric constants of tephroite, fayalite and olivine and the oxide additivity rule, Phys. Chem. Miner., 18, 1-6, doi:10.1007/BF00199037.

Smyth, J. R., and T. C. McCormick (1995), Crystallographic data for minerals, in Mineral Physics and Crystallography: A Handbook of Physical Constants, edited by T. J. Ahrens, pp. 1-17, AGU, Washington, D. C., doi:10.1029/RF002.

Stoller, R. E. (2012), Primary radiation damage formation, in Comprehensive Nuclear Materials, vol. 1, edited by R. J. M. Konings et al., pp. 293-332, Elsevier, Amsterdam, doi:10.1016/B978-0-08-056033-5.00027-6.

Sueno, S., M. Cameron, and C. T. Prewitt (1976), Orthoferrosilite: High-temperature crystal chemistry, Am. Mineral., 61, 38-53.

Thompson, M. S., R. Christoffersen, T. J. Zega, and L. P. Keller (2014), Microchemical and structural evidence for space weathering in soils from asteroid Itokawa, Earth Planets Space, 66, 89, doi:10.1186/1880-5981-66-89.

Tilocca, A., N. H. de Leeuw, and A. N. Cormack (2006), Shell-model molecular dynamics calculations of modified silicate glasses, Phys. Rev. B: Condens. Matter, 73, 104209, doi:10.1103/PhysRevB.73.104209.

Verma, A. K., and B. B. Karki (2009), Ab initio investigations of native and protonic point defects in $\mathrm{Mg}_{2} \mathrm{SiO}_{4}$ polymorphs under high pressure, Earth Planet. Sci. Lett., 285, 140-149, doi:10.1016/j.epsl.2009.06.009.

Walker, A. M., K. Wright, and B. Slater (2003), A computational study of oxygen diffusion in olivine, Phys. Chem. Miner., 30, 536-545, doi:10.1007/s00269-003-0358-7.

Walker, A. M., S. M. Woodley, B. Slater, and K. Wright (2009), A computational study of magnesium point defects and diffusion in forsterite, Phys. Earth Planet. Inter., 172, 20-27, doi:10.1016/j.pepi.2008.04.001.

Wang, L. M., M. L. Miller, and R. C. Ewing (1993a), HRTEM study of displacement cascade damage in krypton-ion-irradiated silicate-Olivine, Ultramicroscopy, 51, 339-347.

Wang, L. M., W. L. Gong, and R. C. Ewing (1993b), Amorphization and dynamic recovery of $\mathrm{A}_{2} \mathrm{BO}_{4}$ structure types during $1.5 \mathrm{MeV}$ krypton ion-beam irradiation, in Symposium A - Materials Synthesis and Processing Using lon Beams, MRS Online Proceedings Library, vol. 316, edited by R. J. Culbertson et al., pp. 247-252, Cambridge Univ. Press, doi:10.1557/PROC-316-247. 


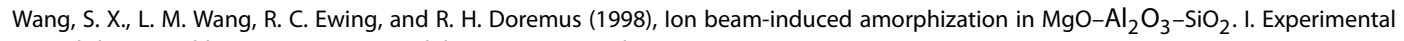
and theoretical basis, J. Non-Cryst. Solids, 238, 198-213, doi:10.1016/S0022-3093(98)00694-2.

Wolf, D., P. Keblinski, S. R. Phillpot, and J. Eggebrecht (1999), Exact method for the simulation of Coulombic systems by spherically truncated, pairwise $r^{-1}$ summation, J. Chem. Phys., 110, 8254-8282, doi:10.1063/1.478738.

Yamada, M., S. Sasaki, H. Nagahara, A. Fujiwara, S. Hasegawa, H. Yano, T. Hiroi, H. Ohashi, and H. Otake (1999), Simulation of space weathering of planet-forming materials: Nanosecond pulse laser irradiation and proton implantation on olivine and pyroxene samples, Earth Planets Space, 51, 1255-1265, doi:10.1186/BF03351599.

Yang, C., E. Zarkadoula, M. T. Dove, I. T. Todorov, T. Geisler, V. V. Brazhkin, and K. Trachenko (2014), Solid-state diffusion in amorphous zirconolite, J. Appl. Phys., 116, 184901, doi:10.1063/1.4901326.

Zarkadoula, E., R. Devanathan, W. J. Weber, M. A. Seaton, I. T. Todorov, K. Nordlund, M. T. Dove, and K. Trachenko (2014), High-energy radiation damage in zirconia: Modeling results, J. Appl. Phys., 115, 083507, doi:10.1063/1.4866989. 